\title{
The Smart-Insole Dataset: Gait Analysis Using Wearable Sensors with a Focus on Elderly and Parkinson's Patients
}

\author{
Chariklia Chatzaki ${ }^{1, *}$, Vasileios Skaramagkas ${ }^{2} \mathbb{1}$, Nikolaos Tachos ${ }^{3,4}$, Georgios Christodoulakis ${ }^{2}$, \\ Evangelia Maniadi ${ }^{1}$, Zinovia Kefalopoulou ${ }^{5}$, Dimitrios I. Fotiadis ${ }^{3,4}$ and Manolis Tsiknakis ${ }^{1,2}$
}

1 Biomedical Informatics and eHealth Laboratory, Department of Electrical and Computer Engineering, Hellenic Mediterranean University, Estavromenos, 71004 Heraklion, Greece; emaniadi@hmu.gr (E.M.); tsiknaki@hmu.gr (M.T.)

2 Computational BioMedicine Laboratory, Institute of Computer Science, Foundation for Research and Technology—Hellas, Vassilika Vouton, 71110 Heraklion, Greece; vskaramag@ics.forth.gr (V.S.); gxristod@ics.forth.gr (G.C.)

3 Unit of Medical Technology and Intelligent Information Systems, Department of Materials Science and Engineering, University of Ioannina, 45110 Ioannina, Greece; ntachos@uoi.gr (N.T.); fotiadis@cs.uoi.gr (D.I.F.)

4 Department of Biomedical Research, Institute of Molecular Biology and Biotechnology, Foundation for Research and Technology-Hellas, 45110 Ioannina, Greece

5 Neurology Department, Patras University Hospital, 26404 Patra, Greece; zkefalopoulou@gmail.com

* Correspondence: rchatzaki@hmu.gr

\section{check for} updates

Citation: Chatzaki, C.; Skaramagkas, V.; Tachos, N.; Christodoulakis, G.; Maniadi, E.; Kefalopoulou, Z.; Fotiadis, D.I.; Tsiknakis, M. The Smart-Insole Dataset: Gait Analysis Using Wearable Sensors with a Focus on Elderly and Parkinson's Patients. Sensors 2021, 21, 2821. https:// doi.org/10.3390/s21082821

Academic Editors: Toshiyo Tamura and Antonio Suppa

Received: 16 February 2021

Accepted: 14 April 2021

Published: 16 April 2021

Publisher's Note: MDPI stays neutral with regard to jurisdictional claims in published maps and institutional affiliations.

Copyright: (c) 2021 by the authors. Licensee MDPI, Basel, Switzerland. This article is an open access article distributed under the terms and conditions of the Creative Commons Attribution (CC BY) license (https:// creativecommons.org/licenses/by/ $4.0 /)$.

\begin{abstract}
Gait analysis is crucial for the detection and management of various neurological and musculoskeletal disorders. The identification of gait events is valuable for enhancing gait analysis, developing accurate monitoring systems, and evaluating treatments for pathological gait. The aim of this work is to introduce the Smart-Insole Dataset to be used for the development and evaluation of computational methods focusing on gait analysis. Towards this objective, temporal and spatial characteristics of gait have been estimated as the first insight of pathology. The Smart-Insole dataset includes data derived from pressure sensor insoles, while 29 participants (healthy adults, elderly, Parkinson's disease patients) performed two different sets of tests: The Walk Straight and Turn test, and a modified version of the Timed Up and Go test. A neurologist specialized in movement disorders evaluated the performance of the participants by rating four items of the MDS-Unified Parkinson's Disease Rating Scale. The annotation of the dataset was performed by a team of experienced computer scientists, manually and using a gait event detection algorithm. The results evidence the discrimination between the different groups, and the verification of established assumptions regarding gait characteristics of the elderly and patients suffering from Parkinson's disease.
\end{abstract}

Keywords: gait analysis; Parkinson's disease; insoles; pressure sensors; dataset

\section{Introduction}

Gait is a rhythmic, periodic movement that requires coordination, balance, and synchronization, activated by the proper functioning of the central and peripheral (musculoskeletal) nervous system. Movement is powered by the ground reaction forces (GRF) applied to the body through its contact with the ground. The physical and psychological state of a person significantly differentiates the gait characteristics, creating a unique pattern for each person [1]. However, several typical patterns of gait can be detected that relate to normal, nonpathological gait. Accurate gait analysis is crucial for a variety of systems that support clinical experts in the diagnosis and management of patients that present pathological gait. Even more, it can provide evidence for the recognition of falls, which are common in older people [2].

To analyze gait, the gait cycle is employed, which is identical to stride and includes two successive steps. The phases describing a gait cycle are: (a) the stance phase that occupies 
$60 \%$ of the gait cycle and (b) the swing phase that occupies $40 \%$ of the gait cycle [3-5]. The stance phase begins with the first contact of the foot with the ground and ends when the same foot leaves the ground, while the swing phase begins with the foot to leave the ground and ends with the next contact of the same foot with the ground. A more detailed breakdown of stance and swing phases into periods, namely: initial contact, loading response, mid-stance, terminal stance, pre-swing, mid-swing, and terminal swing, has been proposed in the literature in order to achieve a better understanding of gait [6,7]. For the analysis of a gait cycle, the recognition of the characteristic events of gait that describe the position/contact of the foot (heel and toe), with respect to the ground, is needed. In a normal gait cycle, the sequence of events that takes place is the Heel Strike, which indicates the beginning of stance, followed by the Foot Flat and Heel Rise, until the Toe Off event appears, which indicates the beginning of the swing phase, and ends with the next Heel Strike. The sequence of the characteristic gait events and the correlation of the different subdivisions of gait phases (events, periods, and phases) can be seen in Figure 1. Typically, force plates are used as sensing systems for measuring the GRF during gait, which provide accurate analysis. Nevertheless, force plates are relatively high-priced, require expert operation and are limited to laboratory settings [8]. The use of wearable sensors has the advantage of transferring gait analysis out of the laboratory in daily life. Towards this direction, sensor insoles for measuring foot pressure is one of the current state-of-the-art technologies used for gait analysis since each gait event can be described with a pressure pattern, and thus, phases and gait patterns can be analyzed [9]. Alternatively, inertial measurement units (IMUs) and electromyography (EMG) signals are widely exploited to power algorithms for discriminating the gait phases and patterns [10]. Combinations of the aforementioned sensory systems are also used [6]. In this work, pressure sensor insoles are exploited for the generation of the Smart-Insole dataset, as an appropriate solution for gait analysis in real-life settings, with the focus on elderly and Parkinson's disease patients.

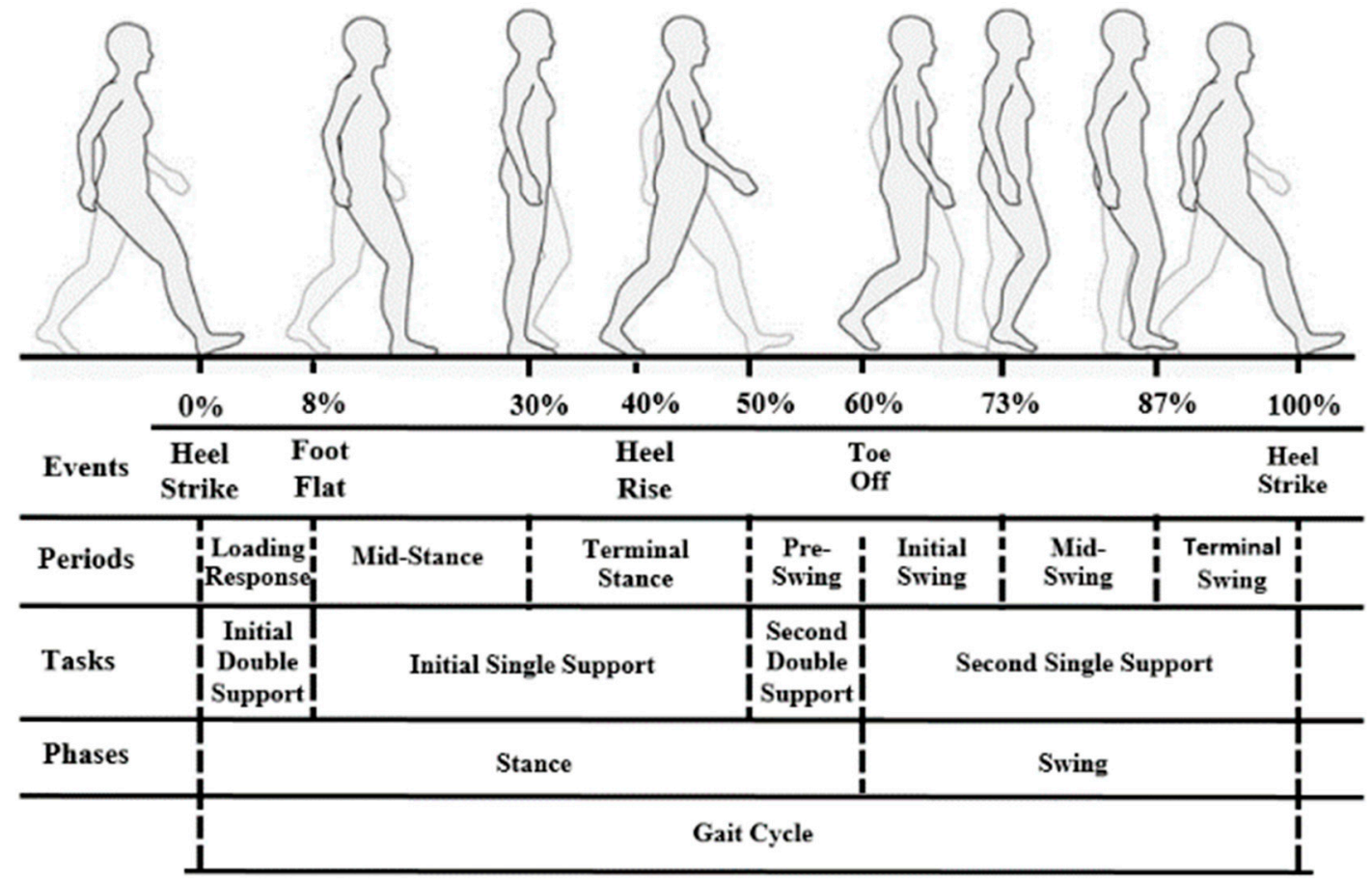

Figure 1. The gait cycle: events, periods, tasks, and phases. (Figure designed based on [6,11-13]).

\section{The Smart-Insole Dataset}

Smart-Insole is a publicly available dataset (available for download from https: / / bmi.hmu.gr (accessed on 14 April 2021) focused on gait analysis, which includes data from a pair of pressure sensors insoles where 29 participants from 3 different groups of 
interest (healthy adults, elderly, Parkinson's disease patients), performed two sets of tests. The protocol of the Smart-Insole study has received ethical approval from the Hellenic Mediterranean University Research Ethics Committee (Approval number: 9/01.04.2020). The dataset's generation details regarding the equipment, the measurement protocol, the undertaking tests, the participant's details, and the following annotation process are described in Sections 2.1-2.3.

\subsection{Data Acquisition Details}

For the development of the Smart-Insole Dataset, the Moticon SCIENCE [14] pressure sensor insole was selected (Figure 2). This choice was based on the following facts: (a) the power supply, the storage, and the data transmission unit are integrated into the insole; (b) the system has a sufficient number (16) of pressure sensors; (c) it includes a 6-Axis Inertial Measurement Unit (IMU) sensor for acceleration and angular rate data. The insole system has been validated in terms of functionality and accuracy of the data it provides [15-17]. For the needs of the recordings, a lightweight and flexible pair of shoes was purchased in which the insole was fitted.
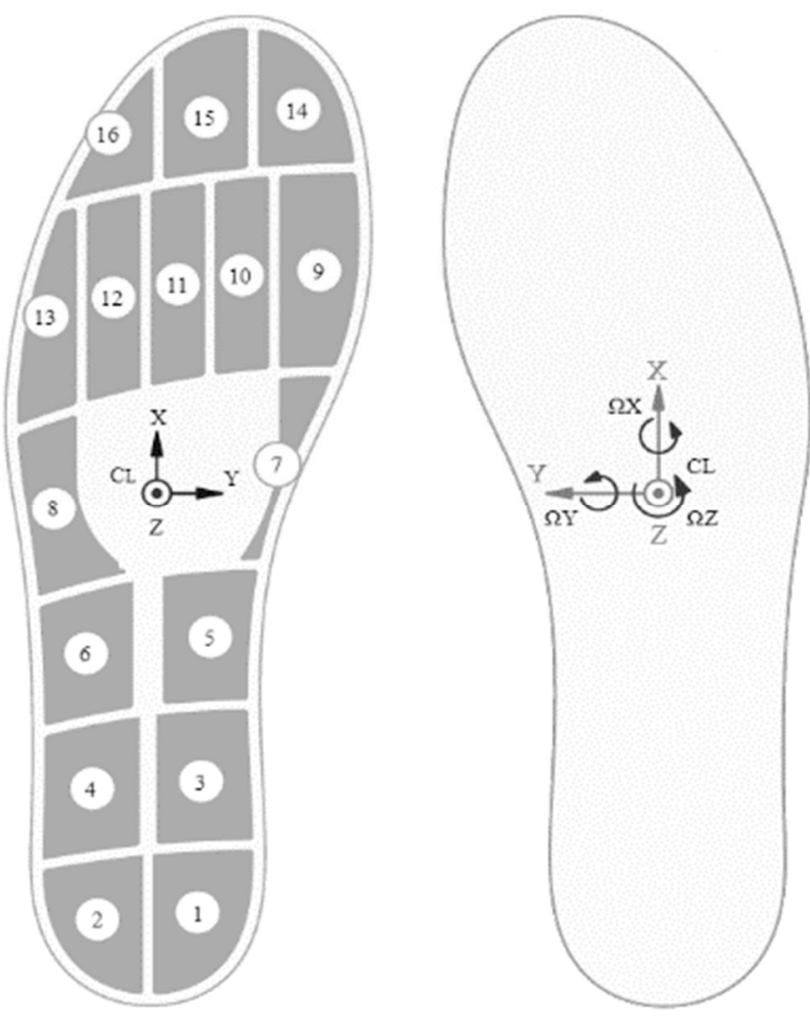

Figure 2. The Moticon SCIENCE pressure sensor insole (model insole 3). Reproduced with permission from Moticon ReGo AG [14].

The sampling rate was set at $100 \mathrm{~Hz}$. The generated file for each recording includes 51 features in total, 25 values for the left and 25 values for the right leg, plus the timestamp:

1. The timestamp (ms)

2. The pressure from 1 to 16 sensors $\left(\mathrm{N} / \mathrm{cm}^{2}\right)$

3. The acceleration in the $x, y, z$ axes $(g)$

4. The angular rate in $\omega_{\mathrm{x}}, \omega_{\mathrm{y}}, \omega_{\mathrm{z}}(\mathrm{dps})$

5. The computed center of pressure in the $x, y$ coordinates $(-0.5 \ldots+0.5$ (related to insole length/width))

6. The computed by Moticon, total force (N) 


\subsection{Measurement Protocol Details}

Several measurement protocols have been proposed for the evaluation of gait characteristics, postural stability, as well as risk of falling for people who have a normal or abnormal gait. Most of these protocols include trials with a number of steps in a straight path and at different speeds, walking on a sloped surface and on stairs. In the case of people with an abnormal gait, and especially for patients with Parkinson's disease, the following three tests have been mostly performed: (1) the Timed Up and Go test (TUG test) [18-22], where the participants rise from a sitting position, walk a $3 \mathrm{~m}$ distance, turn, walk back and sit on a chair; (2) walking in a corridor with obstacles, [23-25] and; (3) the Dual-Task test, where the participants walk and at the same time undertake a second process, such as an arithmetic operation, conversation, transferring an object, etc. [26-28].

The Smart-Insole's measurement protocol was developed having in mind the previously described findings and the need for: (a) simplicity, so that it is easily understandable by all groups of participants (healthy adults, elderly, Parkinson's disease patients); (b) safety, so that there is no physical exhaustion or even risk of falling, referring mainly to the elderly and Parkinson's disease patients, and; (c) completeness of the experimental procedures, so that valuable data are recorded for the evaluation of a complete gait analysis system. Items from the part III "Motor examination" of the Movement Disorder Society (MDS)-sponsored revision of the Unified Parkinson's Disease Rating Scale (UPDRS) [29] has been used for the clinical assessment of motor signs of Parkinson's disease and as guidelines for the experimental protocol. Taking all these into consideration, the protocol for the generation of the Smart-Insole dataset includes the Walking Straight and Turn test and a modified version of the Timed Up and Go test, which are described in detail in Sections 2.2.1 and 2.2.2. During the recordings, two action cameras were set along the route (one at the end and one at the middle of the route). The cameras were recording only the lower body part, i.e., the legs of the participants, so that the verification of the recorded raw data during the annotation process would be possible. However, it is worth mentioning that the public version of the dataset includes only the anonymized data containing the raw values from the insole sensors (for which participants have signed consent forms on making them publicly available in the scientific community) and the annotated version of the data as described in Section 2.4.

\subsubsection{The Walk Straight and Turn Test}

In the Walk Straight and Turn (WST) test, participants were requested to walk in a straight route for $10 \mathrm{~m}$ starting from an upright position (standing). At the end of the 10-m route, they turned $180^{\circ}$ and returned to the starting position (Figure 3a). The WST test was organized in line with the description of the item-3.10 "Gait" of the MDS-UPDRS. From a clinical point of view, it provides valuable information for stride amplitude and speed, heel strike, and turning, while it also contributes to the rating of items 3.11 "Freezing of Gait" and 3.13 "Posture". Participants were asked to complete the tests as a normal and continuous set of movements. The test was repeated two times and at three different walking speeds, slow, normal, and fast, as perceived by each participant. Different speeds of walking have been studied for their impact on gait characteristics [30] and on differencing gait between Parkinson's disease patients with mild and moderate severity [31]. For the recordings, the start and endpoints of the route were clearly marked on the floor.

\subsubsection{The modified Timed Up and Go Test}

In the modified Timed Up and Go (TUG) test, participants were instructed to rise from the chair (if possible, without any support of the upper limbs) naturally (without any intended stopping pose) and immediately started walking in a straight path for $10 \mathrm{~m}$. At the end of the $10-\mathrm{m}$ route, they made an $180^{\circ}$ turn and returned to the sitting position (Figure $3 b$ ). In the typical form of TUG test, the walking distance is $3 \mathrm{~m}$ and the participants are rated with a completion time score which indicates their level of physical mobility [18]. In our work, the MDS-UPDRS has been used as a guideline for the clinical examination 
of motor signs of Parkinson's Disease. Thus, in the deployed protocol, we included a modified version of the TUG test to obtain data from its initial phase, i.e., the getting up phase, which align with the 3.9-item "Arising from chair" of the MDS-UPDRS, but we extended the walking distance to $10 \mathrm{~m}$ to obtain more data during walking. The test was repeated twice.

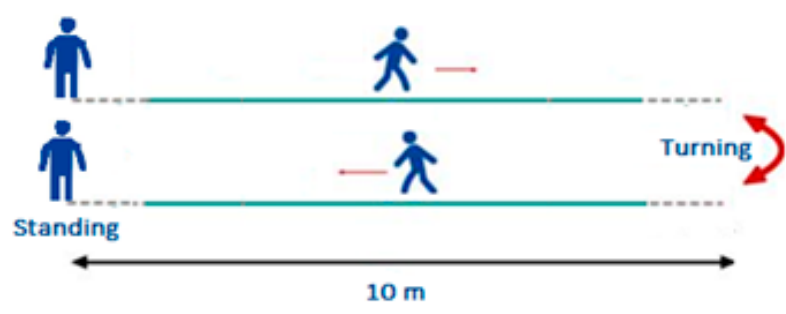

(a)

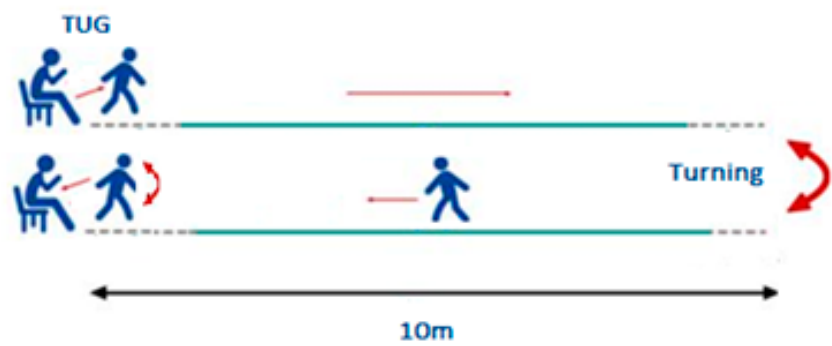

(b)

Figure 3. Left figure (a) The Walking Straight Test, and right figure (b) The Timed Up and Go test.

\subsection{Dataset Participants}

The first release of the Smart-Insole dataset includes recordings from 29 participants who were categorized into three groups, as shown in Table 1. The first group is the control group, in which healthy adults aged between $20-59$ years were included. The second group includes elderly citizens, in which people above the age of 60 years were included. For these two groups, volunteers having musculoskeletal or neurological diseases that could affect their gait or balance were excluded from participation. The third group relates to Parkinson's disease patients, irrespective of their age. All participants with Parkinson's disease had received their medication as normally scheduled. For the participants that would find it hard to complete all tests, the number and repetitions were adjusted so that they felt comfortable.

Table 1. The details of Smart-Insole's Dataset participants.

\begin{tabular}{ccccccc}
\hline Group & No. of Recordings & $\begin{array}{c}\text { Average Age } \\
\text { (Years) }\end{array}$ & $\begin{array}{c}\text { Age Span } \\
\text { (Years) }\end{array}$ & $\begin{array}{c}\text { Height } \\
\text { (cm) }\end{array}$ & $\begin{array}{c}\text { Weight } \\
\mathbf{( K g )}\end{array}$ & Gender \\
\hline Healthy adults (S) & 13 & 38 & $20-58$ & 176 & 81 & 2 Females, 11 Males \\
Elderly (EL) & 9 & 74 & $60-85$ & 173 & 80 & All Males \\
PD patients (PD) & 8 & 72 & $63-83$ & 172 & 80 & All Males \\
\hline
\end{tabular}

The public version of the Smart-Insole dataset does not include the data of PD001; thus, although eight participants of the "Parkinson" group are used in the results of this study, the public version of the dataset includes seven recordings.

A Neurologist specialized in movement disorders evaluated the performance of the participants by rating four items from part III "Motor examination" of the MDSUPDRS [32], i.e., item 3.9 "Arising from chair", item-3.10 "Gait", item-3.11 "Freezing of gait", and item-3.14 "Global spontaneity of movement", that match with the measurement protocol followed. In so doing, the neurologist has carefully examined the video recordings obtained by the two cameras (one at the end and one at the middle of the $10 \mathrm{~m}$-route) used during the recordings. Previous works evidence the feasibility of remote assessment of PD patients even via videoconference sessions. Specifically, part III "Motor examination" of the MDS-UPDRS (excluding item-3.3 "Rigidity" and item-3.12 "Postural Stability", which require an in-person assessment), has been evaluated for the assessment of motor signs of PD in several studies [33-37]. The MDS-UPDRS rating for each participant is shown in Table 2. 
Table 2. The rating of four MDS-UPDRS items [29], based on the analysis of participant's videos.

\begin{tabular}{|c|c|c|c|c|}
\hline Participant * & $\begin{array}{l}\text { "MDS-UPDRS-3.9" } \\
\text { Arising from Chair }\end{array}$ & $\begin{array}{l}\text { "MDS-UPDRS-3.10" } \\
\text { Gait }\end{array}$ & $\begin{array}{l}\text { “MDS-UPDRS-3.11" } \\
\text { Freezing of Gait }\end{array}$ & $\begin{array}{l}\text { "MDS-UPDRS-3.14" } \\
\text { Global Spontaneity of Movement }\end{array}$ \\
\hline PD001 & 2 & 3 & 1 & 2 \\
\hline PD002 & 0 & 1 & 0 & 1 \\
\hline PD003 & 4 & 4 & 4 & 4 \\
\hline PD004 & 1 & 1 & 0 & 1 \\
\hline PD005 & 3 & 2 & 1 & 3 \\
\hline PD006 & 0 & 1 & 0 & 1 \\
\hline PD007 & 1 & 2 & 0 & 2 \\
\hline PD008 & 4 & 3 & 0 & 3 \\
\hline EL001 & 0 & 0 & 0 & 0 \\
\hline EL002 & 1 & 1 & 0 & 1 \\
\hline EL003 & 1 & 0 & 0 & 0 \\
\hline EL004 & 1 & 0 & 0 & 0 \\
\hline EL006 & 0 & 0 & 0 & 0 \\
\hline EL007 & 1 & 1 & 0 & 1 \\
\hline EL008 & 0 & 0 & 0 & 0 \\
\hline EL009 & 0 & 0 & 0 & 0 \\
\hline EL010 & 0 & 0 & 0 & 0 \\
\hline S001 & 0 & 0 & 0 & 0 \\
\hline S002 & 0 & 0 & 0 & 0 \\
\hline S003 & 0 & 0 & 0 & 0 \\
\hline S004 & 0 & 0 & 0 & 0 \\
\hline S005 & 0 & 1 & 0 & 0 \\
\hline S006 & 0 & 0 & 0 & 0 \\
\hline S007 & 0 & 0 & 0 & 0 \\
\hline S008 & 0 & 0 & 0 & 0 \\
\hline S009 & 0 & 0 & 0 & 0 \\
\hline S010 & 0 & 0 & 0 & 0 \\
\hline S011 & 0 & 0 & 0 & 0 \\
\hline S012 & 0 & 1 & 0 & 0 \\
\hline S013 & 0 & 0 & 0 & 0 \\
\hline
\end{tabular}

* PDxxx refers to Parkinson's patients, ELxxx refers to elderly, and Sxxx refers to healthy adults participating in the Smart-Insole dataset. The tag EL005 has not been used.

\subsection{Smart-Insole Dataset Annotation}

The labeling of the recorded data was performed following a two-level annotation process. In the first level of annotation, data were described as activities of daily living (ADLs), following the labeling protocol of our previous work "MobiAct" [38], a benchmark dataset for activity and fall recognition. The first level of annotation of ADLs, included 12 different labels (Table 3), which described the sequence of activities undertaken by the participants in the tests. The second level of annotation was focused on labeling the characteristic events of a gait cycle (HES-FOF-HER-TOF-HES). For gait analysis, the labeling of events of both legs was mandatory. In a normal gait cycle, the described sequence starts with the one foot on Heel Strike, and the other foot on Toe Off. Consequently, in the Smart-Insole dataset the labels of four events of gait can be found for the left and right leg as described in Table 4.

For the annotation of the data, we followed a hybrid model of both manual and automated labeling. In each case, to reassure the reliability of data labeling, cross-checks with the signal alternation and the captured videos were performed. The annotation of ADLs was made purely manually, through inspection of signal alternation and the captured video regarding the timestamp. 
Table 3. Activities of daily living (ADLS): annotation of Smart-Insole dataset, Level 1.

\begin{tabular}{lcc}
\hline Label & Activity & Description \\
\hline STD & Standing & Standing with subtle movements \\
STE & Standing Eyes closed & Standing with eyes closed \\
WAL & Walking & Normal walking \\
WAS & Walking Slow & Walking in a slow rhythm \\
WAF & Walking Fast & Walking in a fast rhythm \\
SCH & Sit on chair & Sitting on a chair \\
CHU & Chair up & Getting up from a chair \\
SIT & Sitting & Sitting with subtle movements \\
TUR & Turning & Turning 180 degrees at a normal speed at the end of the $10 \mathrm{~m}$ aisle. \\
TUS & Turning Slow & Turning 180 degrees at a slow speed at the end of the $10 \mathrm{~m}$ aisle. \\
TUF & Turning Fast & Turning 180 degrees at a fast speed at the end of the $10 \mathrm{~m}$ aisle. \\
\hline
\end{tabular}

Table 4. Characteristic events of gait analysis: annotation of Smart-Insole dataset, Level 2.

\begin{tabular}{ccc}
\hline Label & Activity & Description \\
\hline HES & Heel Strike & Heel contacts the floor \\
HER & Heel Rise & Heel rises of the floor \\
TOF & Toe Off & Toe leaves the floor \\
FOF & Foot Flat & Foot is flat on the floor; both heel and toe contact the floor. \\
\hline
\end{tabular}

The automated annotation of gait events was made using a gait event detection algorithm that was developed for this purpose. The results of the algorithm were crossvalidated using the signal and video data. The developed gait event detection algorithm was based on: (a) a modified version (Figure 4) of the core body of knowledge referring to transition between states, of the gait phase detection algorithm developed by Pappas et al. [39]; (b) the pressure threshold parameter (Equation (1)), for heel and toe of both left and right legs; (c) the heel and toe parameters, which store the average values of pressure sensors with respect to the timestamp inquest, and d) the previous state parameter, which holds the previous event of the normal flow of a gait cycle (GC) namely, HES-FOF-HERTOF.

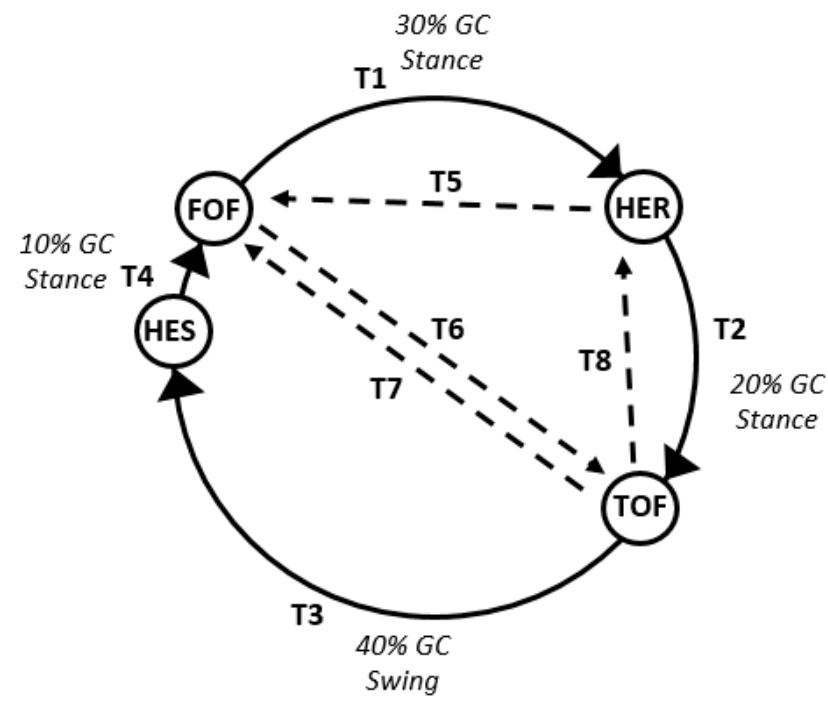

Figure 4. The core workflow of the developed gait event detection algorithm (based on [39] transition of states).

Initially, a pressure detection algorithm, based on Equation (1), was deployed to determine if the deployed pressure exceeded the threshold and, thus, the inquest area of the foot touching the ground. The pressure detection algorithm was based on the 
force detection algorithm, included in the timing analysis module (TAM) of the Tekscan software [40], in a similar manner as deployed in the work of Catalfamo et al. [41]. The only difference is that Catalfamo et al. used a force-based sensing system. In our work, the pressure factor was empirically adjusted, through a continuous test-and-check process, for each participant for the left and right leg separately. The assigned values for the pressure factor span from 0.04-0.12, while in the original work of Catalfamo et al. [36] it was set to 0.1 . The min and max variables of Equation (1) are the minimum and maximum pressures of the recorded data.

$$
\text { Pressure Threshold }=\min +(\max -\min ) \times \text { Pressure Factor } .
$$

We considered that pressure sensors 1 and 2 represented the area of the heel (heel parameter), while sensors 14,15 , and 16, represented the pressure area of the toe (toe parameter), according to the arrangement of sensors shown in Figure 2. Employing more sensors (1-4 for heel and 9-16 for toe) did not affect the results. Thus, it was decided to use the minimum set of sensors.

The core workflow of the developed gait event detection algorithm is shown in Figure 4. It is based on the gait-phase detection methodology followed by Pappas et al. [39,42], who proposed seven transitions of activities between two gait events (Heel Off, Heel Strike) and two phases (Swing, Stance). In our work, we adopt these seven transitions (T1-T7) with the modification that we foresee them as transitions between the four gait events (heel strike, foot flat, heel rise, toe off) of a gait cycle. The sequence of events and its association with the transitions T1-T2-T3-T4, are shown in Figure 4. In the Smart-insole dataset, all tests begin with the participants in the standing or sitting positions and, thus, the gait begins from FOF, in consequence, the first transition T1 refers to the transition of FOF to HER event. Transitions T1, T2, T3, and T4 are expected to be observed in a normal gait cycle. The abnormalities that are most likely to appear, are covered by the transition T5, T6, T7 and T8 (added by our team). In the abnormal transition T5, the foot, instead of following a Toe Off event, and thus getting in to swing phase, moves to Foot Flat state from the Heel Rise, and retaining the stance; in the T6, Toe Off from Foot Flat is observed instead of Heel Rise; and in the T7, the foot is flat on the floor after Toe Off event instead of Heel Strike event. These abnormalities are most likely to appear in the gait of the elderly and in Parkinson's disease patients since they could express uncertainty on the gait, lack of stability, and disorientation. The T8 transition relates to the abnormality of toe walking. Since the Heel Rise event describes a state of the foot where only the toe touches the ground, it can also be seen as a Toe On event. Therefore, the T8 transition describes a loop of Toe On and Toe Off events. Toe walking is mainly seen in children [43]. It has neither been observed in the Smart-Insole dataset nor is expected to be observed in the target population under study. It is, however, described for reasons of completeness. Data were labeled with the name of the event taking place until a new one is identified. If abnormalities are observed, then the previous event is also reported in the annotated version of the dataset. For both normal and abnormal transitions, the swing phase is considered to start when the Toe Off event occurs and to end when a new event takes place (T3, T7, T8), while in all other cases the stance phase prevails (T1, T2, T4, T5, and T6). The condition checks and the respective labels of the developed gait event detection algorithm for labeling are shown in Table 5. 
Table 5. The condition checks of the developed gait event detection algorithm for labeling.

\begin{tabular}{|c|c|}
\hline Label & Condition Check \\
\hline HES & $\begin{array}{l}(\text { Heel }>\text { Force Threshold Heel }) \wedge(\text { Toe } \leq \text { Force Threshold Toe }) \wedge \\
(\text { Previous State }=\text { TOF })\end{array}$ \\
\hline FOF & $\begin{array}{l}(\text { Heel }>\text { Force Threshold Heel }) \wedge(\text { Toe }>\text { Force Threshold Toe }) \wedge \\
(\text { Previous State }=\text { HES })\end{array}$ \\
\hline HER & $\begin{array}{l}(\text { Heel } \leq \text { Force Threshold Heel }) \wedge(\text { Toe }>\text { Force Threshold Toe }) \wedge \\
(\text { Previous State }=\text { FOF })\end{array}$ \\
\hline TOF & $\begin{array}{l}(\text { Heel } \leq \text { Force Threshold Heel }) \wedge(\text { Toe } \leq \text { Force Threshold Toe }) \wedge \\
(\text { Previous State }=\text { HER })\end{array}$ \\
\hline FOF (from HER) & $\begin{array}{l}(\text { Heel }>\text { Force Threshold Heel }) \wedge(\text { Toe }> \\
\text { Force Threshold Toe }) \wedge(\text { Previous State }=\text { HER })\end{array}$ \\
\hline TOF (from FOF) & $\begin{array}{l}(\text { Heel } \leq \text { Force Threshold Heel }) \wedge(\text { Toe } \leq \text { Force Threshold Toe }) \wedge \\
(\text { Previous State }=\text { FOF })\end{array}$ \\
\hline FOF (from TOF) & $\begin{array}{l}(\text { Heel }>\text { Force Threshold Heel }) \wedge(\text { Toe }>\text { Force Threshold Toe }) \wedge \\
(\text { Previous State }=\text { FOF })\end{array}$ \\
\hline HER (from TOF) & $\begin{array}{l}(\text { Heel } \leq \text { Force Threshold Heel }) \wedge(\text { Toe }>\text { Force Threshold Toe }) \wedge \\
(\text { Previous State }=\text { TOF })\end{array}$ \\
\hline
\end{tabular}

\section{Gait Analysis Methodology}

The analysis of human body movement includes the notions of kinetics and kinematics. Kinetics describes the causes of movement and focuses on the forces (mass, acceleration), the torques, and the produced power of a movement. In contrast, kinematics focuses on the linear and angular description of the movement (changes in velocity position, displacement, and acceleration, over time). Therefore, gait analysis requires the quantified study of the force parameters, and the time and distance parameters, by calculating important characteristics (temporal and spatial, respectively).

The temporal characteristics can be categorized as purely temporal, phase temporal, or spatiotemporal. The phase-temporal characteristics have been normalized based on the duration of the gait cycle. The spatial characteristics have been calculated following an empirically driven methodology, based on the knowledge of the total distance of the route. For the estimation of the spatiotemporal characteristics data were divided into straight lines and turns, based on the analysis of video recordings and the respective timestamps. Straight lines' data include recordings until the last step of straight walking, while Turns' data include recordings from the first step indicating a change of direction (Toe Off event) until the final return step (Heel Strike event), of participants facing the return route. Therefore, three samples of recordings were analyzed for each participant's record, two for straight-line walking and one during turning. The distance of the route for straight line walking corresponds to $10 \mathrm{~m}, \pm 1$ step since some participants turned slightly before or after the marked $10 \mathrm{~m}$ aisle. Nevertheless, we claim that this does not have a major effect on the results of spatial characteristics, taking into consideration the range of the number of steps number (14.45 to 22.47), shown in Table 6. Performing a cross-check on our calculations (by adjusting \pm 1 step) of the step length (as a key metric for spatial characteristics as given by Equation (13)), we can observe that the effect is limited to the second decimal place most of the time. The distance cover during turning corresponds to $1.5 \mathrm{~m}$, as a mean value of a gait cycle's length (Table 6), which is evidence for both spin-turn and step-turn strategies [44]. For the evaluation of the Smart-Insole dataset, and for the recognition of gait phases and events as a mandatory predecessor of gait pattern recognition, the following gait temporal and spatial characteristics were calculated: 
Table 6. Results on Straight Lines (Mean values along with Standard Deviations) of temporal and spatial characteristics using the Smart-Insole Dataset.

\begin{tabular}{|c|c|c|c|c|c|c|c|c|c|c|c|c|}
\hline \multirow{2}{*}{$\begin{array}{c}\text { Type of Test } \\
\begin{array}{c}\text { Group of } \\
\text { Participants }\end{array}\end{array}$} & \multicolumn{3}{|c|}{ WST Slow } & \multicolumn{3}{|c|}{ WST Normal } & \multicolumn{3}{|c|}{ WST High } & \multicolumn{3}{|c|}{ TUG } \\
\hline & $\mathrm{S}$ & EL & PD & $\mathrm{s}$ & EL & PD & $\mathrm{S}$ & EL & PD & $\mathbf{S}$ & EL & PD \\
\hline $\begin{array}{l}\text { Number of } \\
\text { Recordings }\end{array}$ & 52 & 36 & 14 & 52 & 36 & 14 & 52 & 34 & 14 & 52 & 36 & 22 \\
\hline $\begin{array}{c}\text { Left Step } \\
\text { Duration (s) }\end{array}$ & $0.75 \pm 0.13$ & $0.76 \pm 0.17$ & $0.70 \pm 0.10$ & $0.55 \pm 0.05$ & $0.58 \pm 0.07$ & $0.62 \pm 0.15$ & $0.48 \pm 0.10$ & $0.51 \pm 0.12$ & $0.51 \pm 0.11$ & $0.55 \pm 0.15$ & $0.44 \pm 0.14$ & $0.54 \pm 0.09$ \\
\hline $\begin{array}{l}\text { Right Step } \\
\text { Duration (s) }\end{array}$ & $0.75 \pm 0.14$ & $0.77 \pm 0.15$ & $0.73 \pm 0.14$ & $0.57 \pm 0.06$ & $0.58 \pm 0.08$ & $0.58 \pm 0.06$ & $0.54 \pm 0.09$ & $0.49 \pm 0.12$ & $0.53 \pm 0.07$ & $0.57 \pm 0.10$ & $0.62 \pm 0.14$ & $0.63 \pm 0.18$ \\
\hline $\begin{array}{l}\text { Duration (s) } \\
\text { Step Duration (s) }\end{array}$ & $0.75 \pm 0.13$ & $0.76 \pm 0.14$ & $0.72 \pm 0.09$ & $0.56 \pm 0.04$ & $0.58 \pm 0.05$ & $0.60 \pm 0.09$ & $0.51 \pm 0.06$ & $0.50 \pm 0.05$ & $0.52 \pm 0.06$ & $0.57 \pm 0.08$ & $0.54 \pm 0.03$ & $0.58 \pm 0.10$ \\
\hline Stride Duration (s) & $1.50 \pm 0.26$ & $1.54 \pm 0.28$ & $1.44 \pm 0.20$ & $1.13 \pm 0.08$ & $1.18 \pm 0.10$ & $1.20 \pm 0.20$ & $1.02 \pm 0.13$ & $1.00 \pm 0.09$ & $1.04 \pm 0.12$ & $1.12 \pm 0.16$ & $1.07 \pm 0.08$ & $1.18 \pm 0.23$ \\
\hline Steps Number & $15.70 \pm 2.38$ & $22.47 \pm 5.60$ & $22.28 \pm 5.30$ & $14.45 \pm 1.15$ & $20.11 \pm 5.80$ & $19.57 \pm 4.07$ & $12.75 \pm 1.48$ & $14.91 \pm 1.86$ & $17.07 \pm 3.60$ & $12.78 \pm 1.99$ & $17.61 \pm 3.90$ & $17.30 \pm 5.00$ \\
\hline $\begin{array}{l}\text { Single Support } \\
\text { Time (s) }\end{array}$ & $1.26 \pm 0.11$ & $1.27 \pm 0.24$ & $1.16 \pm 0.24$ & $0.91 \pm 0.10$ & $0.87 \pm 0.08$ & $0.98 \pm 0.24$ & $0.84 \pm 0.12$ & $0.83 \pm 0.08$ & $0.77 \pm 0.12$ & $0.89 \pm 0.13$ & $0.83 \pm 0.05$ & $0.91 \pm 0.23$ \\
\hline $\begin{array}{l}\text { Double Support } \\
\text { Time (s) }\end{array}$ & $0.27 \pm 0.13$ & $0.36 \pm 0.16$ & $0.39 \pm 0.16$ & $0.22 \pm 0.08$ & $0.30 \pm 0.10$ & $0.29 \pm 0.09$ & $0.18 \pm 0.07$ & $0.17 \pm 0.11$ & $0.27 \pm 0.09$ & $0.22 \pm 0.09$ & $0.24 \pm 0.09$ & $0.25 \pm 0.09$ \\
\hline $\begin{array}{l}\text { Stance Time (s) } \\
\text { Swing Time (s) }\end{array}$ & $\begin{array}{l}0.86 \pm 0.17 \\
0.61 \pm 0.10\end{array}$ & $\begin{array}{l}0.93 \pm 0.19 \\
0.57 \pm 0.08\end{array}$ & $\begin{array}{l}0.89 \pm 0.16 \\
0.50 \pm 0.04\end{array}$ & $\begin{array}{l}0.67 \pm 0.06 \\
0.45 \pm 0.04\end{array}$ & $\begin{array}{l}0.72 \pm 0.09 \\
0.42 \pm 0.03\end{array}$ & $\begin{array}{l}0.72 \pm 0.15 \\
0.45 \pm 0.04\end{array}$ & $\begin{array}{l}0.59 \pm 0.11 \\
0.45 \pm 0.04\end{array}$ & $\begin{array}{l}0.56 \pm 0.08 \\
0.45 \pm 0.02\end{array}$ & $\begin{array}{l}0.65 \pm 0.09 \\
0.41 \pm 0.07\end{array}$ & $\begin{array}{l}0.63 \pm 0.08 \\
0.43 \pm 0.04\end{array}$ & $\begin{array}{l}0.63 \pm 0.01 \\
0.40 \pm 0.05\end{array}$ & $\begin{array}{l}0.77 \pm 0.43 \\
0.40 \pm 0.04\end{array}$ \\
\hline Single Support (\%) & $86.49 \pm 10.26$ & $83.45 \pm 8.77$ & $81.94 \pm 11.88$ & $\begin{array}{l}0.45 \pm 0.04 \\
80.64 \pm 7.15\end{array}$ & $\begin{array}{l}0.42 \pm 0.03 \\
76.62 \pm 8.57\end{array}$ & $\begin{array}{l}0.45 \pm 0.04 \\
82.72 \pm 8.78\end{array}$ & $\begin{array}{l}0.45 \pm 0.04 \\
81.54 \pm 8.02\end{array}$ & $\begin{array}{c}0.45 \pm 0.02 \\
82.74 \pm 11.05\end{array}$ & $\begin{array}{l}0.41 \pm 0.07 \\
73.27 \pm 8.17\end{array}$ & $\begin{array}{l}0.43 \pm 0.04 \\
82.99 \pm 9.07\end{array}$ & $\begin{array}{c}0.40 \pm 0.05 \\
79.91 \pm 8.10\end{array}$ & $\begin{array}{l}0.40 \pm 0.04 \\
78.82 \pm 8.17\end{array}$ \\
\hline Double Support (\%) & $16.76 \pm 7.86$ & $21.91 \pm 7.57$ & $20.92 \pm 10.34$ & $19.82 \pm 6.45$ & $25.90 \pm 7.39$ & $24.06 \pm 4.10$ & $17.94 \pm 5.40$ & $16.04 \pm 9.12$ & $25.97 \pm 9.00$ & $20.15 \pm 7.45$ & $23.25 \pm 6.88$ & $21.99 \pm 7.72$ \\
\hline Stance Phase $(\%)$ & $58.26 \pm 3.37$ & $61.74 \pm 2.81$ & $63.69 \pm 3.04$ & $59.88 \pm 1.79$ & $62.86 \pm 3.10$ & $61.48 \pm 2.95$ & $56.57 \pm 4.01$ & $55.21 \pm 3.85$ & $61.09 \pm 5.25$ & $59.21 \pm 2.78$ & $61.21 \pm 2.79$ & $63.84 \pm 6.01$ \\
\hline Swing Phase (\%) & $41.74 \pm 3.37$ & $38.26 \pm 2.81$ & $36.31 \pm 3.04$ & $40.12 \pm 1.79$ & $37.14 \pm 3.10$ & $38.52 \pm 2.95$ & $43.45 \pm 4.01$ & $44.79 \pm 3.85$ & $38.91 \pm 5.25$ & $40.79 \pm 2.78$ & $38.79 \pm 2.79$ & $36.16 \pm 6.01$ \\
\hline Gait Velocity $(\mathrm{m} / \mathrm{s})$ & $0.99 \pm 0.20$ & $0.77 \pm 0.26$ & $0.71 \pm 0.20$ & $1.39 \pm 0.17$ & $1.21 \pm 0.34$ & $1.00 \pm 0.29$ & $1.79 \pm 0.28$ & $1.72 \pm 0.35$ & $1.31 \pm 0.35$ & $1.67 \pm 0.37$ & $1.47 \pm 0.36$ & $1.25 \pm 0.45$ \\
\hline Step Length $(\mathrm{m})$ & $0.70 \pm 0.11$ & $0.56 \pm 0.14$ & $0.50 \pm 0.12$ & $0.75 \pm 0.06$ & $0.68 \pm 0.16$ & $0.56 \pm 0.11$ & $0.87 \pm 0.14$ & $0.82 \pm 0.12$ & $0.65 \pm 0.12$ & $0.88 \pm 0.18$ & $0.75 \pm 0.16$ & $0.70 \pm 0.35$ \\
\hline $\begin{array}{l}\text { Step Frequency } \\
\text { (steps/min) }\end{array}$ & $76.67 \pm 12.43$ & $63.21 \pm 9.11$ & $67.59 \pm 8.14$ & $98.18 \pm 7.17$ & $72.47 \pm 6.24$ & $74.19 \pm 8.09$ & $80.00 \pm 8.76$ & $79.68 \pm 7.23$ & $83.21 \pm 4.01$ & $100.67 \pm 12.24$ & $71.45 \pm 8.14$ & $65.18 \pm 19.87$ \\
\hline $\begin{array}{c}\text { Walk Ratio } \\
\text { (mm/step/min) }\end{array}$ & $9.46 \pm 2.65$ & $8.16 \pm 2.48$ & $6.69 \pm 2.41$ & $7.68 \pm 0.78$ & $8.49 \pm 2.56$ & $6.63 \pm 1.27$ & $9.55 \pm 3.21$ & $10.68 \pm 2.17$ & $8.12 \pm 1.53$ & $9.07 \pm 3.37$ & $13.14 \pm 3.46$ & $26.02 \pm 53.65$ \\
\hline
\end{tabular}


- $\quad$ The Step Time (s), which is described as the time between two successive Heel Strikes of different foot.

$$
\text { Step Time }=\text { Heel Strike } \text { H }_{+1}-\text { Heel Strike }_{j} .
$$

- $\quad$ The Stride Time (s), which is equal to the time between two successive Heel Strikes of the same foot.

$$
\text { Stride Time }=\text { Heel Strike } \text { T }_{+2}-\text { Heel Strike }_{j}
$$

- $\quad$ The Stance Time (s, \%), which describes the total time during a gait cycle where the foot is in contact with the ground. Specifically, it is described as the time where the heel of one foot, contacts the ground until the toe of the same foot leaves the ground.

$$
\begin{gathered}
\text { Stance Time }=\text { Toe Off } f_{j+1}-\text { Heel Strike } \\
\text { Stance Phase }(\%)=\left(\frac{\text { Stance Time }}{\text { Gait Cycle }}\right) \times 100 \% .
\end{gathered}
$$

- The Single Support Time (s, \%), which describes the time from the Toe Off of the one foot until the Heel Strike of the other foot.

$$
\begin{gathered}
\text { Single Support Time }=\left(\text { Heel Strike }_{j}-\text { Toe Off }_{j-1}\right)+\left(\text { Heel Strike }_{j+1}-\text { Toe Off }_{j}\right), \\
\text { Single Support Phase }(\%)=\left(\frac{\text { Single Support Time }}{\text { Gait Cycle }}\right) \times 100 \% .
\end{gathered}
$$

- The Double Support Time (s, \%), which describes the time from the Heel Strike of the one foot until the Toe Off of the other foot.

$$
\begin{gathered}
\text { Double Support Time }=\left(\text { Toe Off }_{j}-\text { Heel Strike }_{j}\right)+\left(\text { Toe Off }_{j-1}-\text { Heel Strike }_{j-1}\right), \\
\text { Double Support Phase }(\%)=\left(\frac{\text { Double Support Time }}{\text { Gait Cycle }}\right) \times 100 \%
\end{gathered}
$$

- $\quad$ The Swing Time (s, \%), which describes the time from the Toe Off of the one foot until the Heel Strike of the same foot.

$$
\begin{gathered}
\text { Swing Time }=\text { Heel Strike } j+1-\text { Toe Off } f_{j} \\
\text { Swing Phase }(\%)=\left(\frac{\text { Swing Time }}{\text { Gait Cycle }}\right) \times 100 \% .
\end{gathered}
$$

- The Gait Velocity (m/s), which describes the displacement in the unit of time, is given by the ratio of the total distance to the total time, or by the ratio of the mean values of stride length to stride time.

$$
\text { Gait Velocity }=\frac{\text { Stride Length }}{\text { Stride Time }}
$$

- $\quad$ The Step Length $(\mathrm{m})$ is calculated by dividing the total distance covered $(20 \mathrm{~m})$ to the total number of steps (Steps Number) which is specified as the number of Heel Strikes during gait.

$$
\text { Step Lenght }=\frac{\text { Distance }}{\text { Steps Number }}
$$

- The Stride Length $(\mathrm{m})$ is calculated by dividing the total distance covered $(20 \mathrm{~m})$ to the total number of strides (Strides Number).

$$
\text { Stride Lenght }=\frac{\text { Distance }}{\text { Strides Number }} \text {. }
$$


- The Step Frequency (steps/min) also called cadence or walking rate, describes the number of steps in the unit of time. It is given by the ratio of the steps number to the time of gait, multiplied by 60 to be expressed in minutes.

$$
\text { Step Frequency }=\frac{\text { Steps Number }}{\text { Time }} \times 60 \text {. }
$$

- The Walk Ratio (mm/step/min) represents the relationship between the width (base of gait) and the frequency of steps and is given by the ratio of step length to Step Frequency.

$$
\text { Walk Ratio }=\frac{\text { Step Length }}{\text { Step Frequency }} .
$$

\section{Results}

The experimental results of the temporal and spatial characteristics calculated, are shown in Table 6 for straight-line walking, and in Table 7 for turning. All results and related parameters correspond to mean values and their respective standard deviations, while the distance covered equals $10 \mathrm{~m}$ for straight lines and $1.5 \mathrm{~m}$ for turns. The results are presented according to the type of tests performed and the different groups of participants. The results for WST normal test and the TUG test could be grouped since both represent the self-selected normal pace of walking, and the parts that have been analyzed match. However, we present them separately for potential future comparison. From the SmartInsole dataset, the participant of the Parkinson's patient group with the code number PD008, was excluded from the calculations since he needed a walker to even stand in an upright position. For safety and comfort reasons, one elderly participant completed only one WST at high speed, while two of the Parkinson's patients did not complete all the repetitions or tests. The number of recordings exploited for each group of participants and tests, can be found in Table 6 for straight-line walking and in Table 7 for turning. The reported results and standard deviations have been rounded to two decimal places, and thus, slight deviations may be found in cross-checks. Furthermore, the $p$-values of the estimated results are reported in Tables 8 and 9, to examine the statistical significance of the discrimination between the different groups of participants. 
Table 7. Results on Turns (mean values along with standard deviations) of temporal and spatial characteristics using the Smart-Insole dataset.

\begin{tabular}{|c|c|c|c|c|c|c|c|c|c|c|c|c|}
\hline \multirow{2}{*}{$\begin{array}{c}\text { Type of Test } \\
\begin{array}{c}\text { Group of } \\
\text { Participants }\end{array}\end{array}$} & \multicolumn{3}{|c|}{ WST Slow } & \multicolumn{3}{|c|}{ WST Normal } & \multicolumn{3}{|c|}{ WST High } & \multicolumn{3}{|c|}{ TUG } \\
\hline & $S$ & EL & PD & $\mathrm{S}$ & EL & PD & $S$ & EL & PD & $S$ & EL & PD \\
\hline $\begin{array}{l}\text { Number of } \\
\text { Recordings }\end{array}$ & 26 & 18 & 7 & 26 & 18 & 7 & 26 & 17 & 7 & 26 & 18 & 11 \\
\hline $\begin{array}{c}\text { Left Step } \\
\text { Duration (s) }\end{array}$ & $0.77 \pm 0.23$ & $0.80 \pm 0.25$ & $0.77 \pm 0.28$ & $0.59 \pm 0.16$ & $0.60 \pm 0.09$ & $0.62 \pm 0.12$ & $0.52 \pm 0.16$ & $0.56 \pm 0.17$ & $0.58 \pm 0.13$ & $0.61 \pm 0.17$ & $0.45 \pm 0.25$ & $0.65 \pm 0.24$ \\
\hline $\begin{array}{l}\text { Right Step } \\
\text { Duration (s) }\end{array}$ & $0.79 \pm 0.30$ & $0.88 \pm 0.38$ & $0.70 \pm 0.48$ & $0.59 \pm 0.21$ & $0.63 \pm 0.16$ & $0.50 \pm 0.18$ & $0.58 \pm 0.13$ & $0.50 \pm 0.17$ & $0.49 \pm 0.20$ & $0.58 \pm 0.11$ & $0.63 \pm 0.17$ & $0.81 \pm 0.79$ \\
\hline Step Duration (s) & $0.78 \pm 0.19$ & $0.85 \pm 0.22$ & $0.69 \pm 0.22$ & $0.60 \pm 0.11$ & $0.61 \pm 0.01$ & $0.63 \pm 0.15$ & $0.55 \pm 0.11$ & $0.52 \pm 0.08$ & $0.60 \pm 0.16$ & $0.59 \pm 0.09$ & $0.56 \pm 0.08$ & $0.72 \pm 0.48$ \\
\hline $\begin{array}{l}\text { Stride } \\
\text { Duration (s) }\end{array}$ & $1.56 \pm 0.41$ & $1.69 \pm 0.44$ & $1.49 \pm 0.52$ & $1.19 \pm 0.27$ & $1.24 \pm 0.19$ & $1.13 \pm 0.14$ & $1.11 \pm 0.22$ & $1.06 \pm 0.20$ & $1.08 \pm 0.21$ & $1.19 \pm 0.19$ & $1.09 \pm 0.19$ & $1.46 \pm 0.97$ \\
\hline Steps Number & $3.75 \pm 0.79$ & $4.10 \pm 1.12$ & $4.70 \pm 0.48$ & $3.68 \pm 0.62$ & $3.77 \pm 0.80$ & $4.42 \pm 1.13$ & $4.07 \pm 0.79$ & $4.35 \pm 0.60$ & $4.16 \pm 0.75$ & $3.80 \pm 0.78$ & $4.11 \pm 0.83$ & $4.25 \pm 0.75$ \\
\hline $\begin{array}{c}\text { Single Support } \\
\text { Time (s) }\end{array}$ & $0.97 \pm 0.22$ & $1.17 \pm 0.27$ & $1.01 \pm 0.17$ & $0.88 \pm 0.14$ & $0.88 \pm 0.20$ & $0.92 \pm 0.23$ & $0.79 \pm 0.14$ & $0.86 \pm 0.12$ & $0.81 \pm 0.19$ & $0.89 \pm 0.10$ & $0.76 \pm 0.17$ & $0.98 \pm 0.49$ \\
\hline $\begin{array}{l}\text { Double Support } \\
\text { Time (s) }\end{array}$ & $0.30 \pm 0.24$ & $0.46 \pm 0.26$ & $0.36 \pm 0.28$ & $0.33 \pm 0.14$ & $0.35 \pm 0.13$ & $0.32 \pm 0.11$ & $0.24 \pm 0.14$ & $0.23 \pm 0.13$ & $0.33 \pm 0.18$ & $0.26 \pm 0.11$ & $0.37 \pm 0.15$ & $0.34 \pm 0.23$ \\
\hline $\begin{array}{l}\text { Stance Time (s) } \\
\text { Swing Time (s) }\end{array}$ & $\begin{array}{l}0.73 \pm 0.25 \\
0.61 \pm 0.11\end{array}$ & $\begin{array}{l}0.91 \pm 0.31 \\
0.57 \pm 0.08\end{array}$ & $\begin{array}{l}0.73 \pm 0.23 \\
0.50 \pm 0.04\end{array}$ & $\begin{array}{l}0.62 \pm 0.12 \\
0.45 \pm 0.04\end{array}$ & $\begin{array}{l}0.69 \pm 0.11 \\
0.42 \pm 0.03\end{array}$ & $\begin{array}{l}0.63 \pm 0.10 \\
0.45 \pm 0.04\end{array}$ & $\begin{array}{l}0.55 \pm 0.11 \\
0.45 \pm 0.04\end{array}$ & $\begin{array}{l}0.55 \pm 0.09 \\
0.45 \pm 0.02\end{array}$ & $\begin{array}{l}0.63 \pm 0.09 \\
0.43 \pm 0.04\end{array}$ & $\begin{array}{l}0.60 \pm 0.10 \\
0.44 \pm 0.05\end{array}$ & $\begin{array}{l}0.64 \pm 0.10 \\
0.40 \pm 0.05\end{array}$ & $\begin{array}{l}0.82 \pm 0.61 \\
0.43 \pm 0.13\end{array}$ \\
\hline $\begin{array}{c}\text { Single } \\
\text { Support (\%) }\end{array}$ & $73.54 \pm 14.96$ & $74.66 \pm 12.17$ & $81.75 \pm 13.24$ & $79.89 \pm 12.67$ & $72.70 \pm 12.13$ & $76.16 \pm 12.52$ & $79.29 \pm 12.23$ & $80.46 \pm 8.04$ & $71.16 \pm 13.90$ & $80.35 \pm 8.07$ & $68.75 \pm 13.52$ & $74.40 \pm 9.75$ \\
\hline $\begin{array}{l}\text { Double } \\
\text { Support (\%) }\end{array}$ & $18.19 \pm 13.64$ & $26.40 \pm 11.75$ & $17.89 \pm 15.19$ & $25.65 \pm 12.97$ & $31.78 \pm 10.60$ & $23.73 \pm 9.56$ & $21.05 \pm 12.32$ & $21.61 \pm 10.34$ & $25.71 \pm 18.01$ & $21.11 \pm 8.48$ & $33.84 \pm 12.29$ & $28.88 \pm 10.07$ \\
\hline $\begin{array}{l}\text { Stance Phase (\%) } \\
\text { Swing Phase }(\%)\end{array}$ & $\begin{array}{l}53.62 \pm 7.72 \\
46.38 \pm 7.72\end{array}$ & $\begin{array}{l}60.47 \pm 5.97 \\
39.53 \pm 5.97\end{array}$ & $\begin{array}{l}58.48 \pm 5.64 \\
41.52 \pm 5.64\end{array}$ & $\begin{array}{l}57.43 \pm 4.51 \\
42.57 \pm 4.51\end{array}$ & $\begin{array}{l}61.80 \pm 3.57 \\
38.20 \pm 3.57\end{array}$ & $\begin{array}{l}58.41 \pm 2.21 \\
41.59 \pm 2.21\end{array}$ & $\begin{array}{l}54.65 \pm 4.89 \\
45.35 \pm 4.89\end{array}$ & $\begin{array}{l}54.52 \pm 4.00 \\
45.48 \pm 4.00\end{array}$ & $\begin{array}{l}59.26 \pm 3.74 \\
40.74 \pm 3.74\end{array}$ & $\begin{array}{l}57.49 \pm 3.87 \\
42.51 \pm 3.87\end{array}$ & $\begin{array}{l}61.12 \pm 4.56 \\
38.88 \pm 4.56\end{array}$ & $\begin{array}{l}62.86 \pm 11.57 \\
37.14 \pm 11.57\end{array}$ \\
\hline $\begin{array}{l}\text { Gait Velocity } \\
(\mathrm{m} / \mathrm{s})\end{array}$ & $0.93 \pm 0.38$ & $0.78 \pm 0.27$ & $0.67 \pm 0.20$ & $1.28 \pm 0.40$ & $1.16 \pm 0.25$ & $1.14 \pm 0.33$ & $1.19 \pm 0.32$ & $1.17 \pm 0.50$ & $1.21 \pm 0.51$ & $1.12 \pm 0.29$ & $1.24 \pm 0.45$ & $0.94 \pm 0.43$ \\
\hline $\begin{array}{l}\text { Step Length }(\mathrm{m}) \\
\text { Stride Length }(\mathrm{m})\end{array}$ & $\begin{array}{l}0.57 \pm 0.18 \\
1.35 \pm 0.35\end{array}$ & $\begin{array}{l}0.54 \pm 0.17 \\
1.26 \pm 0.38\end{array}$ & $\begin{array}{l}0.41 \pm 0.06 \\
0.96 \pm 0.37\end{array}$ & $\begin{array}{l}0.59 \pm 0.14 \\
1.44 \pm 0.21\end{array}$ & $\begin{array}{l}0.58 \pm 0.15 \\
1.42 \pm 0.24\end{array}$ & $\begin{array}{l}0.48 \pm 0.15 \\
1.29 \pm 0.37\end{array}$ & $\begin{array}{l}0.52 \pm 0.14 \\
1.30 \pm 0.34\end{array}$ & $\begin{array}{l}0.46 \pm 0.10 \\
1.19 \pm 0.38\end{array}$ & $\begin{array}{l}0.50 \pm 0.14 \\
1.25 \pm 0.39\end{array}$ & $\begin{array}{l}0.57 \pm 0.16 \\
1.33 \pm 0.32\end{array}$ & $\begin{array}{l}0.52 \pm 0.14 \\
1.29 \pm 0.35\end{array}$ & $\begin{array}{l}0.49 \pm 0.14 \\
1.19 \pm 0.39\end{array}$ \\
\hline $\begin{array}{l}\text { Step Frequency } \\
\text { (steps/min) }\end{array}$ & $62.28 \pm 15.00$ & $59.32 \pm 18.72$ & $72.95 \pm 17.26$ & $73.72 \pm 15.67$ & $73.99 \pm 16.91$ & $70.87 \pm 17.20$ & $86.30 \pm 20.78$ & $89.49 \pm 13.29$ & $73.22 \pm 14.27$ & $74.53 \pm 12.37$ & $79.61 \pm 12.67$ & $72.67 \pm 17.52$ \\
\hline $\begin{array}{c}\text { Walk Ratio } \\
\text { (mm/step/min) }\end{array}$ & $10.18 \pm 5.71$ & $10.48 \pm 5.62$ & $6.00 \pm 2.03$ & $8.59 \pm 3.44$ & $8.60 \pm 3.88$ & $7.38 \pm 3.67$ & $6.74 \pm 3.42$ & $5.38 \pm 1.83$ & $7.33 \pm 3.42$ & $8.05 \pm 3.28$ & $6.93 \pm 3.18$ & $8.17 \pm 7.00$ \\
\hline
\end{tabular}




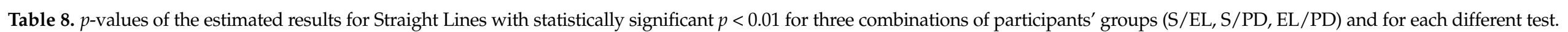

\begin{tabular}{|c|c|c|c|c|c|c|c|c|c|c|c|c|}
\hline \multirow{2}{*}{$\begin{array}{c}\text { Type of Test } \\
\text { Groups Compared }\end{array}$} & \multicolumn{3}{|c|}{ WST Slow } & \multicolumn{3}{|c|}{ WST Normal } & \multicolumn{3}{|c|}{ WST High } & \multicolumn{3}{|c|}{ TUG } \\
\hline & S/EL & S/PD & EL/PD & S/EL & S/PD & EL/PD & S/EL & S/PD & EL/PD & S/EL & S/PD & EL/PD \\
\hline Recordings per Group & $52 / 36$ & $52 / 14$ & $36 / 14$ & $52 / 36$ & $52 / 14$ & $36 / 14$ & $52 / 36$ & $52 / 14$ & $36 / 14$ & $52 / 36$ & $52 / 14$ & $36 / 14$ \\
\hline Left Step Duration (s) & 0.53 & 0.29 & 0.22 & $<0.01$ & $<0.01$ & 0.29 & 0.29 & 0.39 & 0.99 & $<0.01$ & 0.81 & $<0.01$ \\
\hline Right Step Duration (s) & 0.50 & 0.71 & 0.43 & 0.42 & 0.72 & 0.81 & 0.01 & 0.72 & 0.19 & 0.02 & 0.06 & 0.92 \\
\hline Step Duration (s) & 0.56 & 0.38 & 0.22 & 0.01 & 0.02 & 0.49 & 0.36 & 0.51 & 0.17 & 0.08 & 0.37 & 0.01 \\
\hline Stride Duration (s) & 0.49 & 0.43 & 0.24 & 0.01 & 0.02 & 0.50 & 0.34 & 0.60 & 0.15 & 0.08 & 0.25 & 0.01 \\
\hline Single Support Time (s) & 0.82 & 0.02 & 0.15 & 0.06 & 0.09 & 0.01 & 0.86 & 0.09 & 0.05 & 0.02 & 0.57 & 0.05 \\
\hline Double Support Time (s) & $<0.01$ & $<0.01$ & 0.63 & $<0.01$ & $<0.01$ & 0.62 & 0.47 & $<0.01$ & $<0.01$ & 0.16 & 0.18 & 0.77 \\
\hline Stance Time (s) & 0.08 & 0.54 & 0.52 & $<0.01$ & 0.04 & 0.86 & 0.21 & 0.07 & $<0.01$ & 0.96 & 0.03 & 0.06 \\
\hline Single Support (\%) & 0.15 & 0.15 & 0.62 & 0.01 & 0.36 & 0.03 & 0.56 & $<0.01$ & $<0.01$ & 0.10 & 0.07 & 0.63 \\
\hline Double Support (\%) & $<0.01$ & 0.10 & 0.71 & $<0.01$ & 0.02 & 0.38 & 0.23 & $<0.01$ & $<0.01$ & 0.05 & 0.35 & 0.53 \\
\hline Stance Phase (\%) & $<0.01$ & $<0.01$ & 0.03 & $<0.01$ & 0.01 & 0.16 & 0.12 & $<0.01$ & $<0.01$ & $<0.01$ & $<0.01$ & 0.02 \\
\hline Swing Phase (\%) & $<0.01$ & $<0.01$ & 0.03 & $<0.01$ & 0.01 & 0.16 & 0.12 & $<0.01$ & $<0.01$ & $<0.01$ & $<0.01$ & 0.02 \\
\hline Gait Velocity (m/s) & $<0.01$ & $<0.01$ & 0.44 & $<0.01$ & $<0.01$ & 0.05 & 0.31 & $<0.01$ & $<0.01$ & 0.01 & $<0.01$ & 0.04 \\
\hline Step Length (m) & $<0.01$ & $<0.01$ & 0.16 & $<0.01$ & $<0.01$ & 0.01 & 0.07 & $<0.01$ & $<0.01$ & $<0.01$ & $<0.01$ & 0.47 \\
\hline Stride Length (m) & $<0.01$ & $<0.01$ & 0.14 & $<0.01$ & $<0.01$ & 0.02 & 0.08 & $<0.01$ & $<0.01$ & $<0.01$ & 0.03 & 0.75 \\
\hline $\begin{array}{l}\text { Step Frequency } \\
\text { (steps/min) }\end{array}$ & $<0.01$ & 0.01 & 0.12 & $<0.01$ & $<0.01$ & 0.42 & 0.85 & 0.18 & 0.09 & $<0.01$ & $<0.01$ & 0.10 \\
\hline $\begin{array}{c}\text { Walk Ratio } \\
(\mathrm{mm} /(\mathrm{step} / \mathrm{min})\end{array}$ & 0.02 & $<0.01$ & 0.06 & 0.03 & $<0.01$ & 0.01 & 0.07 & 0.11 & $<0.01$ & $<0.01$ & 0.02 & 0.15 \\
\hline
\end{tabular}




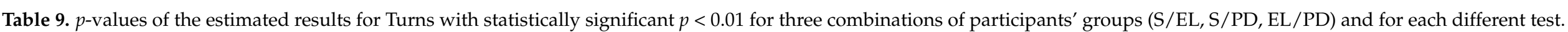

\begin{tabular}{|c|c|c|c|c|c|c|c|c|c|c|c|c|}
\hline \multirow{2}{*}{$\begin{array}{c}\text { Type of Test } \\
\text { Group of Participants }\end{array}$} & \multicolumn{3}{|c|}{ WST Slow } & \multicolumn{3}{|c|}{ WST Normal } & \multicolumn{3}{|c|}{ WST High } & \multicolumn{3}{|c|}{ TUG } \\
\hline & S/EL & S/PD & EL/PD & S/EL & S/PD & EL/PD & S/EL & S/PD & EL/PD & S/EL & S/PD & EL/PD \\
\hline Number of Recordings & $26 / 18$ & $26 / 7$ & $18 / 7$ & $26 / 18$ & $26 / 7$ & $18 / 7$ & $26 / 18$ & $26 / 7$ & $18 / 7$ & $26 / 18$ & $26 / 7$ & $18 / 7$ \\
\hline Left Step Duration (s) & 0.72 & 0.97 & 0.85 & 0.90 & 0.66 & 0.59 & 0.55 & 0.44 & 0.75 & 0.01 & 0.64 & 0.04 \\
\hline Right Step Duration (s) & 0.35 & 0.57 & 0.36 & 0.47 & 0.34 & 0.10 & 0.08 & 0.21 & 0.98 & 0.24 & 0.13 & 0.33 \\
\hline Step Duration (s) & 0.30 & 0.21 & 0.11 & 0.96 & 0.55 & 0.59 & 0.36 & 0.32 & 0.09 & 0.28 & 0.18 & 0.18 \\
\hline Stride Duration (s) & 0.34 & 0.66 & 0.35 & 0.51 & 0.60 & 0.19 & 0.43 & 0.77 & 0.81 & 0.08 & 0.17 & 0.11 \\
\hline Steps Number & $<0.01$ & 0.71 & 0.14 & 0.94 & 0.59 & 0.65 & 0.08 & 0.77 & 0.41 & $<0.01$ & 0.38 & 0.09 \\
\hline Single Support Time (s) & 0.02 & 0.55 & 0.34 & 0.54 & 0.84 & 0.50 & 0.94 & 0.17 & 0.16 & $<0.01$ & 0.12 & 0.70 \\
\hline Double Support Time (s) & 0.03 & 0.97 & 0.17 & 0.06 & 0.79 & 0.24 & 0.92 & 0.07 & 0.06 & 0.19 & 0.07 & 0.21 \\
\hline Swing Time (s) & 0.95 & 0.20 & 0.20 & 0.06 & 0.49 & 0.53 & 0.73 & 0.16 & 0.05 & $<0.01$ & 0.05 & 0.22 \\
\hline Single Support (\%) & 0.05 & 0.88 & 0.15 & 0.10 & 0.71 & 0.09 & 0.87 & 0.45 & 0.50 & $<0.01$ & 0.01 & 0.25 \\
\hline Double Support (\%) & $<0.01$ & 0.16 & 0.44 & $<0.01$ & 0.58 & 0.02 & 0.92 & 0.03 & 0.01 & 0.00 & 0.03 & 0.56 \\
\hline Stance Phase (\%) & $<0.01$ & 0.16 & 0.44 & $<0.01$ & 0.58 & 0.02 & 0.92 & 0.03 & 0.01 & 0.00 & 0.03 & 0.56 \\
\hline Swing Phase (\%) & 0.10 & 0.08 & 0.43 & 0.27 & 0.39 & 0.83 & 0.89 & 0.87 & 0.86 & 0.31 & 0.13 & 0.08 \\
\hline Gait Velocity $(\mathrm{m} / \mathrm{s})$ & 0.73 & 0.03 & 0.07 & 0.80 & 0.07 & 0.14 & 0.14 & 0.74 & 0.47 & 0.27 & 0.14 & 0.60 \\
\hline Step Length (m) & 0.25 & 0.01 & 0.12 & 0.73 & 0.15 & 0.30 & 0.34 & 0.76 & 0.74 & 0.73 & 0.25 & 0.44 \\
\hline Stride Length (m) & 0.41 & 0.11 & 0.10 & 0.95 & 0.68 & 0.68 & 0.57 & 0.15 & 0.01 & 0.19 & 0.70 & 0.21 \\
\hline $\begin{array}{l}\text { Step Frequency } \\
\text { (steps/min) }\end{array}$ & 0.63 & 0.07 & 0.04 & 0.99 & 0.42 & 0.48 & 0.14 & 0.70 & 0.08 & 0.26 & 0.94 & 0.51 \\
\hline
\end{tabular}


Focusing firstly on the different tests and groups, we notice that the different speeds of gait significantly affected the estimated gait characteristics. Changes were observed among others on the step length (Figure 5), the duration of stance (Figure 6), and swing (Figure 7) phases, and were mainly noted in the analysis of straight-line walking. Once someone increases his/her gait velocity, it is expected that the step length and the swing will increase, while the stance will decrease. Taking as baseline the WST normal, which represents a self-selected normal pace by participants, these changes are observed in all groups when moving to the WST high in straight-line walking, in which participants increased their pace of gait. It is worth mentioning that the mean values of the height of the participants (Table 1) differ only slightly and, thus, the effect of this parameter is only minimal. An important aspect to highlight is that \% of stance and swing phases decrease and increase, respectively, during straight-line walking in the case of reduction of gait velocity (WST Slow). In this case, the step length also decreases (Figures 5-7). The change can also be observed in the walking ratio for all groups. The ratio increases both when slowing down (WST Slow) and speeding up (WST high), as shown in Figure 8. These observations on slow walking are in line with previously published results $[45,46]$. Regarding the TUG test, increased values for the walk ratio, the step length, the step frequency, as well as, the stance time and phase were observed because of the participation of PD005. The specific patient exhibited a walking pattern of dragging his feet, something that affects the estimation of the respective metrics, and their standard deviations. The stride parameters are affected in a similar manner to the step parameters. The step frequency increases proportionally with the gait velocity (Figure 9), as expected. The duration of stance and swing phases (60\%, 40\% of the total gait cycle) were verified when examining the results of WST Normal and TUG test, which were recorded in normal velocity for the group of healthy adults (S), with $\sim 61 \%$ and $\sim 39 \%$ duration, respectively. Regarding the duration of the left and right steps, which for normal gait are expected to be equal, it was verified for the majority of the results on all groups. One should always keep in mind that: a) the \pm 0.1 differences are due to rounding of decimals, b) the differences that appeared for the $S$ group, especially in the WST high test were due to the recording of one participant (S008) in which a highly disproportional gait was observed ( $0.3 \mathrm{~s}$ for the left and $0.6 \mathrm{~s}$ for the right step). This deviation, which is expected in pathological gait, is indeed apparent in the WST High test for the group of Parkinson's patients.

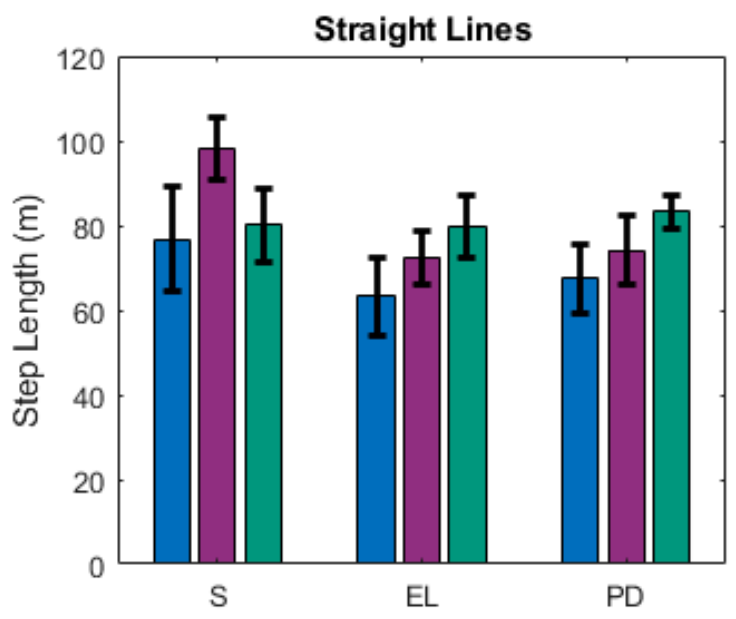

Groups of participants and different speeds of WST

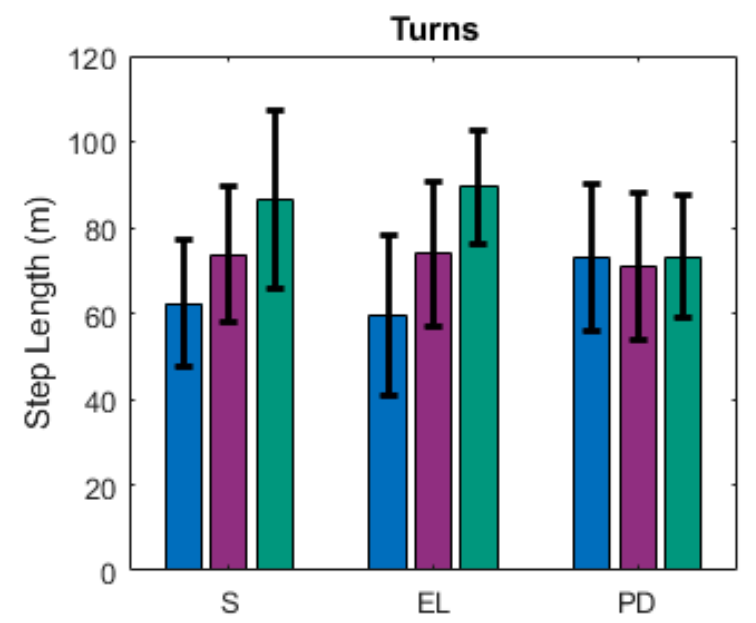

Groups of participants and different speeds of WST

\begin{tabular}{|l|l|l|}
\hline & \\
\hline
\end{tabular}

Figure 5. Changes of the Step Length $(\mathrm{m})$ for Straight Lines and Turns (mean values along with standard deviations) between the different groups of participants (S, EL, PD) and the different speeds of the WST test (slow, normal, high). 

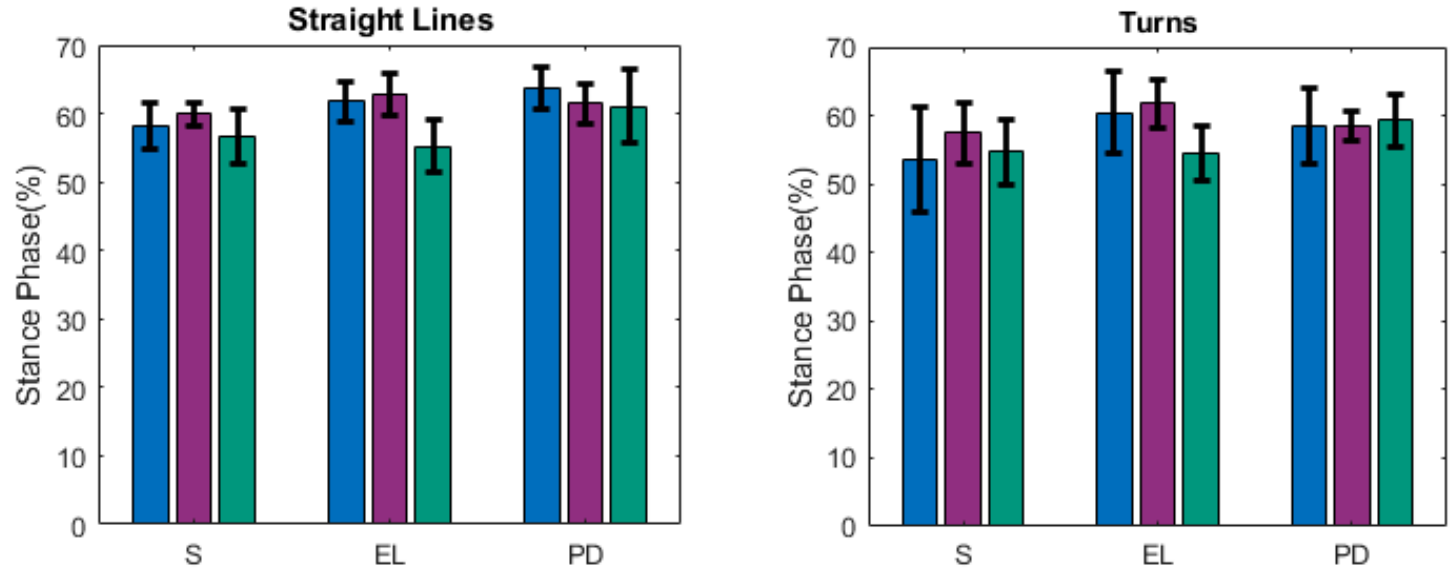

Groups of participants and different speeds of WST

Groups of participants and different speeds of WST

\section{WST Slow $\square$ WST Normal $\square$ WST High I Standard Deviation}

Figure 6. Changes of the Stance Phase (\%) for Straight Lines and Turns (mean values along with standard deviations) between the different groups of participants (S, EL, PD) and the different speeds of the WST test (slow, normal, high).
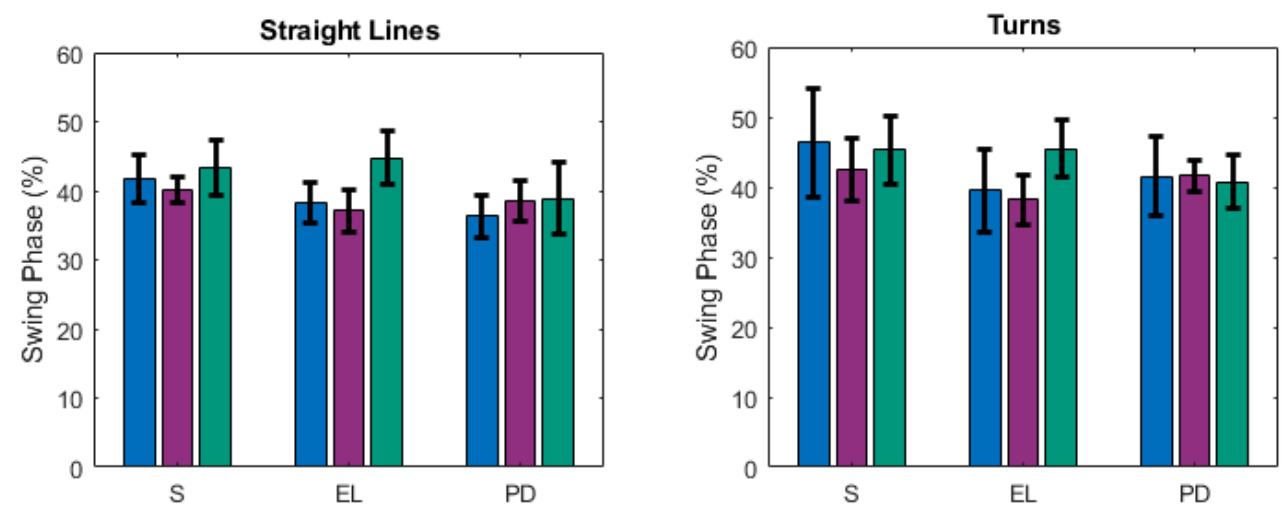

Groups of participants and different speeds of WST

Groups of participants and different speeds of WST

$\square$ wST Slow $\square$ wST Normal $\square$ wST High I Standard Deviation

Figure 7. Changes of the Swing Phase (\%) for Straight Lines and Turns (mean values along with standard deviations) between the different groups of participants (S, EL, PD) and the different speeds of the WST test (slow, normal, high).

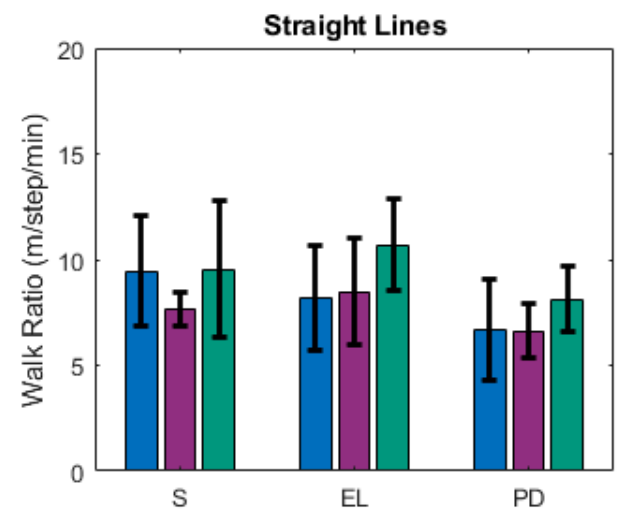

Groups of participants and different speeds of WST

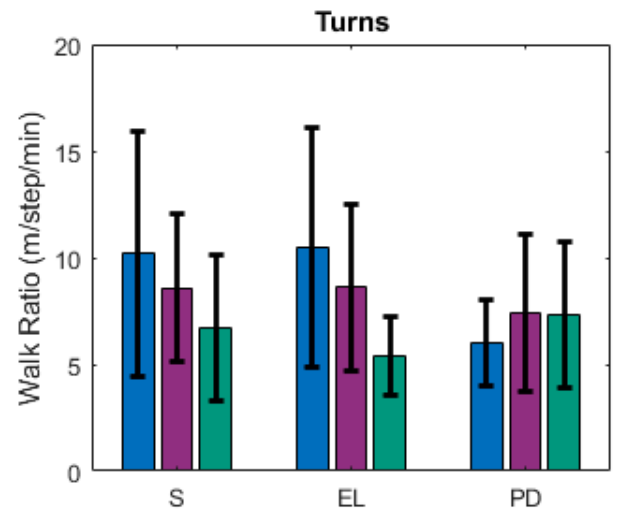

Groups of participants and different speeds of WST

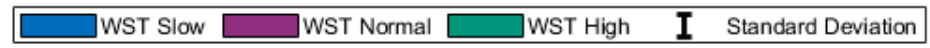

Figure 8. Changes of the Walk Ratio (m/step/min) for Straight Lines and Turns (mean values along with standard deviations) between the different groups of participants (S, EL, PD) and the different speeds of the WST test (slow, normal, high). 

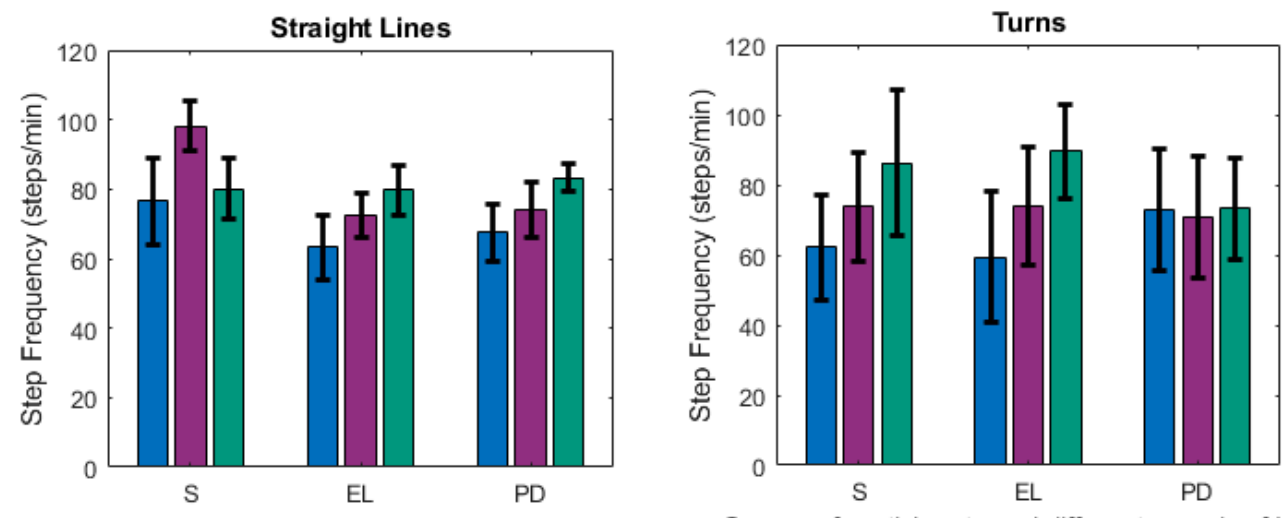

Groups of participants and different speeds of WST

Groups of participants and different speeds of WST

WST Slow $\square$ WST Normal

WST High I Standard Deviation

Figure 9. Changes of the Step Frequency (steps/min) for Straight Lines and Turns (mean values along with standard deviation) between the different groups of participants (S, EL, PD) and the different speeds of the WST test (slow, normal, high).

These results reaffirm the importance of studying different velocities of gait for best revealing gait abnormalities. Furthermore, it is noted that the number of steps, the stance duration, and the double support duration increased for each test as we moved from healthy adults (normal values) to elderly (increased values) and finally to Parkinson's disease patients. Finally, the low standard deviations of the results indicate that data are nicely clustered around the means. Although a low standard deviation is not necessarily required, it is evidence of the fact that all groups exhibited similar behaviors.

In demonstrating the ability of the estimated spatiotemporal characteristics to discriminate between the different groups of participants, when all three levels of speeds for the WST test and the TUG test are taken into consideration, statistical analysis has been performed by estimating the $p$-values of the results (Table 8 , for Straight Lines, and Table 9 for Turns). The following combinations of participants' groups were tested for discrimination for each test: (a) healthy adults (S) versus elderly (EL); (b) healthy adults (S) versus Parkinson's disease patients (PD); and (c) elderly (EL) versus Parkinson's disease patients (PD). The calculated probabilities show clear discrimination, when examining the results on straight-line walking, with statistically significant $p<0.01$ for (a) the S-EL groups, for the WST slow, the WST normal, and the TUG tests; (b) the S-PD groups, for all tests; and (c) the EL-PD groups for the WST high test. Regarding the probabilities on turning data, we cannot state discrimination of the different groups; however, the $p$-values range in many cases from $p<0.01$ to $p=0.05$.

\section{Discussion}

Several datasets suitable for gait analysis, which have been created using sensor insoles [47,48], IMUs [30,49], or pressure and force plates [50,51], are publicly available. However, several concerns exist, with the most important one being the lack of evaluation and/or annotation of the data by specialized neurologists. A second shortcoming is the fact that only the Gait in Parkinson's disease dataset [47] and the dataset proposed by Kluge et al. [30] include data from Parkinson's disease patients. Although the Gait in Parkinson's disease dataset includes a sufficient number (93) of participating Parkinson's disease patients, it only includes recordings of their normal-pace walking. On the other hand, the dataset produced by Kluge et al. [30], includes recordings of Parkinson's disease patients walking with different speeds but includes a very small number (4) of participants.

It is known and most studied for the elderly that different speeds of walking have an impact on the estimated results of spatial and temporal characteristics of gait $[52,53]$. Although limited work has been done in this domain focusing on Parkinson disease patients $[30,54]$, it has been evidenced that short distance walking speed tests can be used 
to discriminate differences in gait function between persons with mild and moderate PD severity [31]. This evidence, together with the observations from our work, as described in Section 4, aligns with the suggestions of $\mathrm{Wu}$ et al. [45] to study the regressions between different gait speeds. These findings suggest that additional work is required for the effect of different levels of walking speed (slow, normal, and fast) on gait analysis.

Critical examination of the MDS-UPDRS ratings of the different groups, shown in Table 2, could lead to a conclusion of a ceiling effect, especially for the groups of elderly and healthy subjects. However, taking into consideration that the exclusion criteria of the protocol used for the groups of elderly and healthy subjects refers to no musculoskeletal or neurological diseases that could affect their gait or balance, this supports our decision to unify the groups from a clinical point of view. Taking a closer look at the groups of elderly and Parkinson's disease patients, this could also be the case for some participants (EL002, EL007, PD002, PD004, and PD006). Nevertheless, these participants have slight gait impairment since they belong to the elderly population (Table 1 shows the average ages of 72 and 74, respectively) irrespective of whether they are Parkinson's disease patients or not. These observations lead to the conclusion/suggestion that for applying computational methods for gait analysis with respect to the MDS-UPDRS, the grouping of participants data should be done not only by age differentiation but also taking their MDS-UPDRS rating into consideration.

These gaps and shortcomings of the available datasets are removed with the release of the Smart-Insole dataset, when used as a dataset for the evaluation of computational methods for detailed gait analysis (exploiting sufficient number of sensors) that can be applied in everyday life (wearable sensor insole), with the focus on elderly people and Parkinson's patients (by adjusting the measurement protocol,) and with the reliability of data annotation provided by a specialized neurologist (rating of four MDS-UPDRS items). However, an increased number of participants would be required to thoroughly examine the gait characteristics of PD patients. A limitation of our study is the lack of a magnetometer, required for the accurate estimation of distance-related parameters. In the present study, these parameters have been approximated using the total distance $(\mathrm{m})$ covered, as described in Section 3.

\section{Conclusions}

The presented Smart-Insole dataset, can be used for the detailed analysis of gait since, to our knowledge, it is the first public gait-related dataset in which the participant's data have been evaluated by a neurologist specialized in movement disorders. It encompasses a fair number of participants of different groups; it is structured in a measurement protocol which is in line with the MDS-UPDRS; and it is developed with the use of wearable sensing equipment as an affordable solution that can be transferred in real life environments. The developed gait event detection algorithm has been double-cross-validated, empirically, by exploiting the recorded video data and, quantifiably by the calculation of the gait characteristics and the verification of established assumptions, as described in Section 4 (propositions of step and stride, stance and swing, left and right step, etc.). The experimental results show clear discrimination between the different groups of users, and the different set of tests performed with different gait speeds.

As this work, has been performed in the context of the Smart-Insole Project, it is the intention of the authors to extend and enrich the dataset: (a) with the use of the current Moticon sensor insole and with the use of a novel smart pressure sensors insole, which is under development in the context of the Smart-Insole project; (b) with the clinical evaluation of the participants by a specialized neurologist using the overall MDS-UPDRS; and (c) with an updated measurement protocol targeting on revealing important cardinal features of Parkinson's disease patients. Finally, the next steps include the deployment of methods for gait pattern recognition and detection. 
Author Contributions: Conceptualization, C.C., N.T., G.C., D.I.F., and M.T.; methodology, C.C., V.S., N.T., G.C., D.I.F., and M.T.; software, C.C. and V.S.; validation, C.C., V.S., and N.T.; formal analysis, C.C., and V.S.; investigation, C.C., N.T., G.C., D.I.F., and M.T.; data curation, C.C., V.S., N.T., and Z.K.; writing-original draft preparation, C.C.; writing-review and editing, C.C., V.S., N.T., G.C., E.M., Z.K., D.I.F., and M.T.; supervision, D.I.F. and M.T. All authors have read and agreed to the published version of the manuscript.

Funding: This research was funded by the European Regional Development Fund of the European Union and Greek national funds through the Operational Program Competitiveness, Entrepreneurship, and Innovation, under the call RESEARCH-CREATE-INNOVATE (project code: T1E $\Delta \mathrm{K}-01888$ ).

Institutional Review Board Statement: The study was conducted according to the guidelines of the Declaration of Helsinki, and approved by the Institutional Ethics Committee of Hellenic Mediterranean University (Approval protocol code and date, 9/01.04.2020).

Informed Consent Statement: Informed consent was obtained from all subjects involved in the study.

Data Availability Statement: The public version of the Smart-Insole Dataset presented in this study (which includes the raw and annotated data from the insole sensors) is available upon request (Biomedical Informatics Laboratory: https://bmi.hmu.gr, accessed on 10 February 2021 or email at: bmi@hmu.gr) for non-commercial, research and educational purposes only, after the sign of a database usage agreement which establish the terms and conditions of data usage.

Acknowledgments: The authors gratefully thank all participants for their contribution in the generation of the Smart-Insole dataset.

Conflicts of Interest: The authors declare no conflict of interest. The funders had no role in the design of the study; in the collection, analyses, or interpretation of data; in the writing of the manuscript, or in the decision to publish the results.

\section{References}

1. Horst, F.; Mildner, M.; Schöllhorn, W.I. One-year persistence of individual gait patterns identified in a follow-up study-A call for individualised diagnose and therapy. Gait Posture 2017, 58, 476-480. [CrossRef]

2. Alkhatib, R.; Diab, M.; Moslem, B.; Corbier, C.; Badaoui, M. El Gait-Ground Reaction Force Sensors Selection Based on ROC Curve Evaluation. J. Comput. Commun. 2015, 3, 13-19. [CrossRef]

3. Whittle, M.W. Gait Analysis-An Intoduction, 4th ed.; Butterworth-Heinemann Ltd.: Oxford, UK, 2007.

4. Müller, B.; Wolf, S.I. Handbook of Human Motion; Springer International Publishing: Cham, Switzerland, 2018 ; ISBN 9783319144177.

5. Wall, J.C.; Charteris, J.; Turnbull, G.I. Two steps equals one stride equals what? The applicability of normal gait nomenclature to abnormal walking patterns. Clin. Biomech. 1987, 2, 119-125. [CrossRef]

6. Taborri, J.; Palermo, E.; Rossi, S.; Cappa, P. Gait partitioning methods: A systematic review. Sensors 2016, 16, 66. [CrossRef] [PubMed]

7. Liu, Y.; Lu, K.; Yan, S.; Sun, M.; Lester, D.K.; Zhang, K. Gait phase varies over velocities. Gait Posture 2014, 39, 756-760. [CrossRef] [PubMed]

8. Chen, S.; Lach, J.; Member, S.; Lo, B.; Member, S. Toward pervasive gait analysis with wearable sensors: A systematic review. IEEE J. Biomed. Health Inform. 2016, 20, 1521-1537. [CrossRef] [PubMed]

9. Díaz, S.; Stephenson, J.B.; Labrador, M.A. Use of wearable sensor technology in gait, balance, and range of motion analysis. Appl. Sci. 2020, 10, 234. [CrossRef]

10. Saboor, A.; Kask, T.; Kuusik, A.; Alam, M.M.; Le Moullec, Y.; Niazi, I.K.; Zoha, A.; Ahmad, R. Latest Research Trends in Gait Analysis Using Wearable Sensors and Machine Learning: A Systematic Review. IEEE Access 2020, 8, 167830-167864. [CrossRef]

11. Vu, H.T.T.; Gomez, F.; Cherelle, P.; Lefeber, D.; Nowé, A.; Vanderborght, B. ED-FNN: A new deep learning algorithm to detect percentage of the gait cycle for powered prostheses. Sensors 2018, 18, 2389. [CrossRef]

12. Neumann, D. Kinesiology of the Musculoskeletal System, 2nd ed.; Mosby: Maryland Heights, MI, USA, 2009; ISBN 978-0323039895.

13. Webster, J.B.; Darter, B.J. 4-Principles of Normal and Pathologic Gait. In Atlas of Orthoses and Assistive Devices, 5th ed.; Elsevier Inc.: Amsterdam, The Netherlands, 2019; pp. 49-62, ISBN 9780323483230.

14. Moticon-SCIENCE. Available online: https://www.moticon.de/ (accessed on 14 December 2020).

15. Braun, B.J.; Veith, N.T.; Hell, R.; Döbele, S.; Roland, M.; Rollmann, M.; Holstein, J.; Pohlemann, T. Validation and reliability testing of a new, fully integrated gait analysis insole. J. Foot Ankle Res. 2015, 8, 54. [CrossRef]

16. Stöggl, T.; Martiner, A. Validation of Moticon's OpenGo sensor insoles during gait, jumps, balance and cross-country skiing specific imitation movements. J. Sports Sci. 2017, 35, 196-206. [CrossRef] 
17. Kakarla, T.P.; Varma, K.A.; Preejith, S.P.; Joseph, J.; Sivaprakasam, M. Accuracy Enhancement of Total Force by Capacitive Insoles. In Medical Measurements and Applications, MeMeA 2019-Symposium Proceedings; Institute of Electrical and Electronics Engineers Inc.: Istanbul, Turkey, 2019.

18. Podsiadlo, D.; Richardson, S. The timed "Up \& Go": A test of basic functional mobility for frail elderly persons. J. Am. Geriatr. Soc. 1991, 39, 142-148. [PubMed]

19. Herman, T.; Giladi, N.; Hausdorff, J.M. Properties of the “Timed Up and Go" test: More than meets the eye. Gerontology 2011, 57, 203-210. [CrossRef]

20. McGrath, D.; Greene, B.R.; Doheny, E.P.; McKeown, D.J.; De Vito, G.; Caulfield, B. Reliability of quantitative TUG measures of mobility for use in falls risk assessment. In Proceedings of the Annual International Conference of the IEEE Engineering in Medicine and Biology Society, EMBS, Boston, MA, USA, 30 August-3 September 2011; Volume 2011, pp. 466-469.

21. Mariani, B.; Jiménez, M.C.; Vingerhoets, F.J.G.; Aminian, K. On-shoe wearable sensors for gait and turning assessment of patients with parkinson's disease. IEEE Trans. Biomed. Eng. 2013, 60, 155-158. [CrossRef] [PubMed]

22. Nieuwboer, A.; De Weerdt, W.; Dom, R.; Peeraer, L.; Lesaffre, E.; Hilde, F.; Baunach, B. Plantar force distribution in Parkinsonian gait: A comparison between patients and age-matched control subjects. Scand. J. Rehabil. Med. 1999, 31, 185-192. [CrossRef]

23. Snijders, A.H.; Weerdesteyn, V.; Hagen, Y.J.; Duysens, J.; Giladi, N.; Bloem, B.R. Obstacle avoidance to elicit freezing of gait during treadmill walking. Mov. Disord. 2010, 25, 57-63. [CrossRef]

24. Moore, S.T.; Yungher, D.A.; Morris, T.R.; Dilda, V.; Macdougall, H.G.; Shine, J.M.; Naismith, S.L.; Lewis, S.J. Autonomous identification of freezing of gait in Parkinson's disease from lower-body segmental accelerometry. J. Neuroeng. Rehabil. $2013,10,1$. [CrossRef]

25. Moon, Y.; Wajda, D.A.; Motl, R.W.; Sosnoff, J.J. Stride-Time Variability and Fall Risk in Persons with Multiple Sclerosis. Mult. Scler. Int. 2015, 2015, 1-7. [CrossRef]

26. Fino, P.C.; Parrington, L.; Pitt, W.; Martini, D.N.; Chesnutt, J.C.; Chou, L.S.; King, L.A. Detecting gait abnormalities after concussion or mild traumatic brain injury: A systematic review of single-task, dual-task, and complex gait. Gait Posture 2018, 62, 157-166. [CrossRef]

27. Jacobs, J.V.; Horak, F.B.; Tran, V.K.; Nutt, J.G. Multiple balance tests improve the assessment of postural stability in subjects with Parkinson's disease. J. Neurol. Neurosurg. Psychiatry 2006, 77, 322-326. [CrossRef]

28. Brauer, S.G.; Woollacott, M.H.; Lamont, R.; Clewett, S.; O'Sullivan, J.; Silburn, P.; Mellick, G.D.; Morris, M.E. Single and dual task gait training in people with Parkinson's Disease: A protocol for a randomised controlled trial. BMC Neurol. 2011, 11, 90. [CrossRef]

29. Goetz, C.G.; Tilley, B.C.; Shaftman, S.R.; Stebbins, G.T.; Fahn, S.; Martinez-Martin, P.; Poewe, W.; Sampaio, C.; Stern, M.B.; Dodel, R.; et al. Movement Disorder Society-Sponsored Revision of the Unified Parkinson's Disease Rating Scale (MDS-UPDRS): Scale presentation and clinimetric testing results. Mov. Disord. 2008, 23, 2129-2170. [CrossRef] [PubMed]

30. Kluge, F.; Gaßner, H.; Hannink, J.; Pasluosta, C.; Klucken, J.; Eskofier, B.M. Towards mobile gait analysis: Concurrent validity and test-retest reliability of an inertial measurement system for the assessment of spatio-temporal gait parameters. Sensors 2017, 17, 1522. [CrossRef]

31. Combs, S.A.; Diehl, M.D.; Filip, J.; Long, E. Short-distance walking speed tests in people with Parkinson disease: Reliability, responsiveness, and validity. Gait Posture 2014, 39, 784-788. [CrossRef]

32. Goetz, C.G.; Fahn, S.; Martinez-Martin, P. The MDS-sponsored Revision of the Unified Parkinson's Disease Rating Scale. J. Mov. Disord. 2008, 1, 1-33.

33. Dorsey, E.R.; Darwin, K.C.; Mohammed, S.; Donohue, S.; Tethal, A.; Achey, M.A.; Ward, S.; Caughey, E.; Conley, E.D.; Eriksson, N.; et al. Virtual research visits and direct-to-consumer genetic testing in Parkinson's disease. Digit. Health 2015, 1. [CrossRef]

34. Dorsey, E.R.; Wagner, J.D.; Bull, M.T.; Rizzieri, A.; Grischkan, J.; Achey, M.A.; Sherer, T.; Chowdhury, S.; Meunier, C.; Cappelletti, L.; et al. Feasibility of virtual research visits in fox trial finder. J. Parkinson's Dis. 2015, 5, 505-515. [CrossRef]

35. Schneider, R.B.; Omberg, L.; Macklin, E.A.; Daeschler, M.; Bataille, L.; Anthwal, S.; Myers, T.L.; Baloga, E.; Duquette, S.; Snyder, P.; et al. Design of a virtual longitudinal observational study in Parkinson's disease (AT-HOME PD). Ann. Clin. Transl. Neurol. 2020, 8, 308-320. [CrossRef]

36. Stillerova, T.; Liddle, J.; Gustafsson, L.; Lamont, R.; Silburn, P. Remotely assessing symptoms of Parkinson's disease using videoconferencing: A feasibility study. Neurol. Res. Int. 2016, 2016, 4802570. [CrossRef] [PubMed]

37. Tarolli, C.; Andrzejewski, K.; Bull, M.; Goldenthal, S.; O’Brien, M.; Simuni, T.; Zimmerman, G.; Biglan, K.; Dorsey, E.R. Virtual research visits in individuals with Parkinson disease enrolled in a clinical trial: REACT-PD Study Interim Analysis (P4.005). Neurology 2017, 88. Available online: http://n.neurology.org/content/88/16_Supplement/P4.005.abstract (accessed on 10 February 2021).

38. Chatzaki, C.; Pediaditis, M.; Vavoulas, G.; Tsiknakis, M. Human Daily Activity and Fall Recognition Using a Smartphone's Acceleration Sensor. In Information and Communication Technologies for Ageing Well and e-Health; Röcker, C., O'Donoghue, J., Ziefle, M., Helfert, M., Molloy, W., Eds.; Springer International Publishing: Cham, Switzerland, 2017; pp. 100-118.

39. Pappas, I.P.I.; Popovic, M.R.; Keller, T.; Dietz, V.; Morari, M. A reliable gait phase detection system. IEEE Trans. Neural Syst. Rehabil. Eng. 2001, 9, 113-125. [CrossRef]

40. Tekscan Research Software User Manual; Tekscan Inc.: Boston, MA, USA, 2004. 
41. Catalfamo, P.; Moser, D.; Ghoussayni, S.; Ewins, D. Detection of gait events using an F-Scan in-shoe pressure measurement system. Gait Posture 2008, 28, 420-426. [CrossRef]

42. Pappas, I.P.I.; Keller, T.; Mangold, S. A reliable, gyroscope based gait phase detection sensor embedded in a shoe insole. Proc. IEEE Sens. 2002, 2, 1085-1088.

43. Alvarez, C.; De Vera, M.; Beauchamp, R.; Ward, V.; Black, A. Classification of idiopathic toe walking based on gait analysis: Development and application of the ITW severity classification. Gait Posture 2007, 26, 428-435. [CrossRef]

44. Hase, K.; Stein, R.B. Turning strategies during human walking. J. Neurophysiol. 1999, 81, 2914-2922. [CrossRef]

45. Wu, A.R.; Simpson, C.S.; van Asseldonk, E.H.F.; van der Kooij, H.; Ijspeert, A.J. Mechanics of very slow human walking. Sci. Rep. 2019, 9, 1-10. [CrossRef] [PubMed]

46. Murakami, R.; Otaka, Y. Estimated lower speed boundary at which the walk ratio constancy is broken in healthy adults. J. Phys. Ther. Sci. 2017, 29, 722-725. [CrossRef] [PubMed]

47. Goldberger, A.L.; Amaral, L.A.N.; Glass, L.; Hausdorff, J.M.; Ivanov, P.C.; Mark, R.G.; Mietus, J.E.; Moody, G.B.; Peng, C.; Stanley, H.E. PhysioBank, PhysioToolkit, and PhysioNet: Components of a new research resource for complex physiologic signals. Circulation 2000, 101, e215-e220. [CrossRef]

48. Shimmer. Available online: http:/ / www.shimmersensing.com/ (accessed on 8 February 2021).

49. Chereshnev, R.; Kertész-Farkas, A. HuGaDB: Human gait database for activity recognition from wearable inertial sensor networks. In Analysis of Images, Social Networks and Texts; Lecture Notes in Computer Science; Springer: Cham, Switzerland, 2017; Volume 10716, pp. 131-141.

50. Zheng, S.; Huang, K.; Tan, T.; Tao, D. A cascade fusion scheme for gait and cumulative foot pressure image recognition. Pattern Recognit. 2012, 45, 3603-3610. [CrossRef]

51. Kobayashi, M.M.Y.; Hida, N.; Nakajima, K.; Fujimoto, M. 2019: AIST Gait Database 2019. Available online: https://unit.aist.go. jp/harc/ExPART/GDB2019.html (accessed on 5 December 2020).

52. Chui, K.K.; Lusardi, M.M. Spatial and temporal parameters of self-selected and fast walking speeds in healthy community-living adults aged 72-98 years. J. Geriatr. Phys. Ther. 2010, 33, 173-178. [CrossRef]

53. Jerome, G.J.; Ko, S.; Kauffman, D.; Studenski, S.A.; Ferrucci, L.; Simonsick, E.M. Gait Characteristics Associated with Walking Speed Decline in Older Adults: Results from the Baltimore Longitudinal Study of Aging; Elsevier Ireland Limited: Shannon, Ireland, 2015; Volume 60.

54. Wahid, F.; Begg, R.K.; Hass, C.J.; Halgamuge, S.; Ackland, D.C. Classification of Parkinson's disease gait using spatial-temporal gait features. IEEE J. Biomed. Health Inform. 2015, 19, 1794-1802. [CrossRef] 